Sharif University of Technology
Scientia Iranica
Transactions E: Industrial Engineering
wCIENTIA

Research Note

\title{
New memory-type control charts for monitoring process mean and dispersion
}

\author{
J.O. Ajadi ${ }^{1}$ and M. Riaz* \\ Department of Mathematics and Statistics, King Fahd University of Petroleum and Minerals, Dhahran 31261, Kingdom of Saudi \\ Arabia. \\ Received 1 September 2015; received in revised form 13 July 2016; accepted 31 October 2016
}

\author{
KEYWORDS \\ Average run length; \\ Max-chart; \\ Normality; \\ Control chart; \\ Location and \\ dispersion parameters.
}

\begin{abstract}
Control chart is widely used to monitor the quality of products of industrial or business processes. Max-CUSUM and Max-EWMA are based on memory-type control charts that monitor the process mean and standard deviation simultaneously. This article introduces four new control charts that monitor the process mean and dispersion simultaneously. The proposed control charting schemes are compared with the existing counterparts including Max-EWMA, Max-CUSUM, SS-EWMA, and SS-CUSUM. A case study is presented for practical considerations using a real dataset.
\end{abstract}

(C) 2017 Sharif University of Technology. All rights reserved.

\section{Introduction}

All the manufacturing and non-manufacturing processes are bound to vary. These process variations consist of two types: natural and special cause variations. The natural variation can be background noise, which cannot be controlled, but the special cause variation is due to many artificial factors like faulty machines, operator mistakes, defective items, and so on. Statistical Process Control (SPC) is a collection of useful techniques that helps to differentiate between the two sources of variations. Control chart is a powerful tool of SPC to detect the special cause variation within a process.

Control chart is divided into two types: memoryless and memory-type charts. Shewhart-type control chart is a memoryless chart because it is based only

1. Present address: Department of System Engineering and Engineering Management, City University of Hong Kong, Kowloon, Hong Kong, China.

*. Corresponding author.

E-mail addresses: ajadi_jimoh2001@yahoo.com (J.O.

Ajadi); riazm@kfupm.edu.sa (M. Riaz)

doi: $10.24200 /$ sci. 2017.4483 on the present information. Shewhart [1] introduced the fundamental control charts to monitor process behaviors in terms of location and dispersion parameters. $\bar{X}$-chart is extensively employed in industries to monitor process meanwhile these are extensively employed in industries to monitor process meanwhile $R, S$, and $S^{2}$ control charts are used for monitoring process dispersion/variability. A major limitation of Shewhart chart is that it is poor in detecting small and moderate shifts in a process parameter. Exponentially Weighted Moving Average (EWMA) and cumulative sum (CUSUM) are the major examples of memorytype control charts. They use both past and present information to detect small and moderate shifts in the process parameters. In EWMA chart, past observations are accounted for, but they are given a smaller weight as they become older; so, EWMA chart applies the utmost weight to the current observations and geometrically decreasing weight to all previous observations. CUSUM chart also gives memory using the information of past history. The basic structures of CUSUM and EWMA were presented by Page [2] and Robert [3], respectively. Some recent literatures that improve both EWMA and CUSUM include: Abbasi and Miller [4], Abbas et al. [5], Abbas et al. [6], Abbas 
et al. [7], Riaz et al. [8], Haq [9], Haq [10], Haq et al. [11], and Zaman et al. [12].

One popular approach to monitoring the process location and dispersion requires designing two different charts separately. This is time-consuming and inconvenient to use two separate charts for the process parameters. An alternative approach is in the form of Max chart developed by Chen and Cheng [13]. This chart can simultaneously monitor both process mean and standard deviation on a single chart, but it has deficiency of detecting small shifts in the process parameters. Xie [14] introduced the Max-EWMA, SSEWMA, EWMA-Max, and EWMA-SC control charts to overcome this challenge. Following the inspiration of Xie [14] for process monitoring, Cheng and Thaga [15] and Thaga [16] developed Max-CUSUM and SS- CUSUM, respectively, which are also memorytype control charts. These charts use both present and previous information about the process; they are more effective in detecting small and moderate shifts than the max-chart.

Four new charts are proposed in this article to aid in detecting smaller shifts rather than the existing memory-type univariate control charts for monitoring both location and dispersion simultaneously. The charts are developed by replacing the statistic that monitors the process dispersion of the Max-EWMA, Max-CUSUM, SS-EWMA, and SS-CUSUM with the three parameters' logarithmic transformation to $S^{2}$, suggested by Castagliola [17].

The structure of the rest of this article is as follows: Section 2 describes different memory-type control charts that monitor both location and dispersion simultaneously. Section 3 provides the design structures of the proposed control charts of this study, and Section 4 offers comparisons of the proposals with the existing counterparts. Section 5 includes a case study for our study purposes; and finally Section 6 concludes the findings of our study.

\section{Memory-type control charts for monitoring process mean and dispersion}

In this section, we provide a brief description of the design structures of some useful charts considered in this study. We focus on the structures of simultaneous control charts. The commonly-used memory-type univariate control charts for simultaneous monitoring of the process mean and dispersion are: Max-EWMA, Max-CUSUM, SS-EWMA, and SS-CUSUM. The details of these control charts are given below one by one.

\subsection{Max-EWMA control chart}

Max-EWMA control chart is a memory-type chart introduced by Xie [14]. It is better at detecting small and moderate shifts than at detecting max-chart.
Let $X_{i}=X_{i 1}, \cdots, X_{i n}, i=1,2,3, \cdots$, represent a sequence of samples of size $n$. The samples are independent and identical. They are normally distributed with $\mu_{0}$ and $\sigma_{0}^{2}$ as location and variability parameters, respectively. Based on this sample information, we have:

$$
\bar{X}_{i}=\left(X_{i 1}+\cdots+X_{i n}\right) / n
$$

and:

$$
S_{i}^{2}=\frac{\sum_{j=1}^{n}\left(X_{i j}-\bar{X}\right)^{2}}{n-1},
$$

denoting mean and variance, respectively, for the $i$ th sample. These statistics are the minimum variance unbiased estimator of $\mu_{0}$ and $\sigma_{0}^{2}$, respectively. Two transformed statistics from $\bar{X}_{i}$ and $\bar{S}_{i}^{2}$ are defined below:

$$
\begin{aligned}
& Z_{i}=\sqrt{n} \frac{\left(\bar{X}-\mu_{0}\right)}{\sigma_{0}}, \\
& Y_{i}=\Phi^{-1}\left\{H\left[\frac{(n-1) S_{i}^{2}}{\sigma_{0}^{2}} ; n-1\right]\right\} .
\end{aligned}
$$

In Eq. (2), $\Phi^{-1}($.$) represents inverse standard normal$ cumulative distribution function and $H(., n-1)$ is a chi-square distribution function with $n-1$ degrees of freedom. Functions $Z_{i}$ and $Y_{i}$ are independent statistics and follow the standard normal distribution. The two EWMA statistics derived from $Z_{i}$ and $Y_{i}$ are given below:

$$
\begin{aligned}
& U_{i}=(1-\lambda) U_{i-1}+\lambda Z_{i}, \\
& V_{i}=(1-\lambda) V_{i-1}+\lambda Y_{i}, \\
& M_{i}=\max \left(\left|U_{i}\right|,\left|V_{i}\right|\right),
\end{aligned}
$$

where $0<\lambda \leq 1$, and the initial values for $U_{i}$ and $V_{i}$ are set to be $U_{0}$ and $V_{0}$, respectively. The highest absolute value $\left(M_{i}\right)$ of both statistics $U_{i}$ and $V_{i}$ is calculated, and it is compared with the Upper Control Limit (UCL) given below in Eq. (6b):

$$
\mathrm{UCL}=E\left(M_{i}\right)+L \sqrt{\operatorname{Var}\left(M_{i}\right)} \text {. }
$$

The expected value and variance of $M_{i}$ are derived through the numerical computation and are given as $E\left(M_{i}\right)=1.12379$ and $\operatorname{Var}\left(M_{i}\right)=0.363381$. Therefore:

$$
\mathrm{UCL}=\sqrt{\frac{\lambda}{(2-\lambda)}}(1.128379+L 0.602811)
$$

The ARL results of Max-EWMA control chart, through simulation, for various shifts in the process mean $(a)$ and dispersion $(b)$ for different sample size $(n)$ are provided in Table 1. Max-EWMA chart is very sensitive to small shifts in the process mean and dispersion when the sample size increases and sensitivity parameter $\lambda$ decreases in value. 
Table 1. ARL values for Max-EWMA control charting schemes at $\mathrm{ARL}_{0}=250$.

\begin{tabular}{|c|c|c|c|c|c|c|c|c|c|c|}
\hline & & & & & $a$ & & & & & \\
\hline & $b$ & 0 & 0.25 & 0.5 & 0.75 & 1 & 1.25 & 1.5 & 1.75 & 2 \\
\hline \multirow{8}{*}{$\begin{array}{l}n=5 \\
L=2.785 \\
\lambda=0.10\end{array}$} & 0.25 & 3.22 & 3.22 & 3.21 & 3.22 & 3.17 & 2.98 & 2.56 & 2 & 2 \\
\hline & 0.5 & 5.9 & 5.91 & 5.78 & 4.85 & 3.73 & 3.03 & 2.55 & 2.07 & 2 \\
\hline & 0.75 & 18.53 & 15.61 & 8.49 & 5.24 & 3.81 & 3.05 & 2.54 & 2.16 & 2.02 \\
\hline & 1 & 250.68 & 24.88 & 8.82 & 5.32 & 3.85 & 3.07 & 2.57 & 2.23 & 2.04 \\
\hline & 1.25 & 17.7 & 13.2 & 7.88 & 5.27 & 3.86 & 3.1 & 2.6 & 2.27 & 2.06 \\
\hline & 1.5 & 7.35 & 6.94 & 5.87 & 4.66 & 3.76 & 3.08 & 2.62 & 2.3 & 2.06 \\
\hline & 1.75 & 4.8 & 4.66 & 4.32 & 3.87 & 3.38 & 2.95 & 2.57 & 2.27 & 2.05 \\
\hline & 2 & 3.61 & 3.59 & 3.45 & 3.23 & 2.96 & 2.69 & 2.44 & 2.21 & 2.02 \\
\hline \multirow{7}{*}{$\begin{aligned} n & =5 \\
L & =3.04 \\
\lambda & =0.2\end{aligned}$} & 0.25 & 2.7 & 2.7 & 2.69 & 2.7 & 2.68 & 2.26 & 2 & 2 & 1.97 \\
\hline & 0.75 & 25.29 & 20.23 & 8.46 & 4.64 & 3.2 & 2.5 & 2.11 & 1.93 & 1.74 \\
\hline & 1 & 250.99 & 31.53 & 8.65 & 4.71 & 3.31 & 2.56 & 2.15 & 1.9 & 1.68 \\
\hline & 1.25 & 17.9 & 12.84 & 7.12 & 4.55 & 3.29 & 2.59 & 2.17 & 1.88 & 1.67 \\
\hline & 1.5 & 6.59 & 6.08 & 5.01 & 3.94 & 3.1 & 2.56 & 2.16 & 1.87 & 1.68 \\
\hline & 1.75 & 4.04 & 3.95 & 3.55 & 3.18 & 2.78 & 2.38 & 2.09 & 1.85 & 1.66 \\
\hline & 2 & 3.02 & 2.97 & 2.83 & 2.63 & 2.42 & 2.15 & 1.97 & 1.78 & 1.61 \\
\hline \multirow{8}{*}{$\begin{aligned} \lambda & =0.2 \\
n & =3 \\
L & =3.04\end{aligned}$} & 0.25 & 4.29 & 4.29 & 4.3 & 4.28 & 3.89 & 3.12 & 2.79 & 2.05 & 2 \\
\hline & 0.5 & 11.34 & 11.21 & 10.11 & 6.48 & 4.36 & 3.29 & 2.68 & 2.21 & 2.02 \\
\hline & 0.75 & 67.43 & 51.27 & 14.95 & 6.89 & 4.45 & 3.35 & 2.7 & 2.3 & 2.06 \\
\hline & 1 & 252.17 & 51.57 & 13.52 & 6.83 & 4.51 & 3.41 & 2.77 & 2.35 & 2.1 \\
\hline & 1.25 & 29.95 & 19.79 & 10.49 & 6.35 & 4.45 & 3.47 & 2.8 & 2.39 & 2.12 \\
\hline & 1.5 & 10.43 & 9.31 & 7.26 & 5.39 & 4.19 & 3.34 & 2.8 & 2.42 & 2.12 \\
\hline & 1.75 & 6.14 & 5.89 & 5.24 & 4.39 & 3.69 & 3.17 & 2.71 & 2.39 & 2.11 \\
\hline & 2 & 4.49 & 4.3 & 3.99 & 3.63 & 3.23 & 2.88 & 2.56 & 2.29 & 2.06 \\
\hline \multirow{8}{*}{$\begin{array}{l}\lambda=0.2 \\
n=4 \\
L=3.04\end{array}$} & 0.25 & 3.2 & 3.21 & 3.2 & 3.21 & 3.06 & 2.83 & 2.06 & 2 & 2 \\
\hline & 0.5 & 7.04 & 7.12 & 6.68 & 4.98 & 3.58 & 2.8 & 2.24 & 2.02 & 1.97 \\
\hline & 0.75 & 37.6 & 29.93 & 10.73 & 5.45 & 3.7 & 2.83 & 2.32 & 2.05 & 1.9 \\
\hline & 1 & 246.68 & 39.13 & 10.46 & 5.51 & 3.77 & 2.88 & 2.38 & 2.06 & 1.86 \\
\hline & 1.25 & 22.59 & 15.62 & 8.4 & 5.26 & 3.73 & 2.93 & 2.4 & 2.08 & 1.86 \\
\hline & 1.5 & 7.91 & 7.23 & 5.8 & 4.51 & 3.51 & 2.86 & 2.42 & 2.09 & 1.85 \\
\hline & 1.75 & 4.82 & 4.65 & 4.15 & 3.66 & 3.14 & 2.67 & 2.35 & 2.07 & 1.84 \\
\hline & 2 & 3.52 & 3.44 & 3.27 & 3 & 2.72 & 2.44 & 2.19 & 1.99 & 1.79 \\
\hline
\end{tabular}

\subsection{Max-CUSUM chart}

The design structure of Max-CUSUM control chart was developed by Cheng and Thaga [15]. The statistics of Eqs. (1) and (2) are integrated in CUSUM statistics to monitor the process mean and variance as they are given in Eqs. (7) to (10):

$$
\begin{aligned}
& C_{i}^{+}=\max \left[0, Z_{i}-k+C_{i-1}^{+}\right], \\
& C_{i}^{-}=\max \left[0,-Z_{i}-k+C_{i-1}^{-}\right],
\end{aligned}
$$

$$
\begin{aligned}
& S_{i}^{+}=\max \left[0, Y_{i}-k+S_{i-1}^{+}\right], \\
& S_{i}^{-}=\max \left[0,-Y_{i}-k+S_{i-1}^{-}\right],
\end{aligned}
$$

where $C_{0}=S_{0}=0$ are the starting points. Reference value $k$ is any real number greater than zero (i.e., $k>$ $0)$. We set the value of $k$ to be 0.5 throughout this article.

Since $Z_{i}$ and $Y_{i}$ are both normally distributed, we can combine the statistics, $C_{i}^{+}, C_{i}^{-}, S_{i}^{+}$, and $S_{i}^{-}$, 
Table 2. ARL values for Max-CUSUM control charting schemes at $\mathrm{ARL}_{0}=250$ and $k=0.50$.

\begin{tabular}{|c|c|c|c|c|c|c|c|c|c|c|}
\hline & \multicolumn{10}{|c|}{$a$} \\
\hline & $b$ & 0 & 0.25 & 0.5 & 0.75 & 1 & 1.25 & 1.5 & 1.75 & 2 \\
\hline & 0.25 & 4.59 & 4.61 & 4.61 & 4.57 & 4.23 & 3.52 & 3 & 2.5 & 2.01 \\
\hline & 0.5 & 10.41 & 10.35 & 9.44 & 6.63 & 4.68 & 3.57 & 2.97 & 2.5 & 2.11 \\
\hline & 0.75 & 57.61 & 41.96 & 13.44 & 7.03 & 4.78 & 3.64 & 2.97 & 2.53 & 2.21 \\
\hline$h=5.07$ & 1 & 250.86 & 48.03 & 13.28 & 7.11 & 4.84 & 3.7 & 3.02 & 2.59 & 2.27 \\
\hline \multirow[t]{7}{*}{$n=3$} & 1.25 & 29.89 & 20.63 & 10.84 & 6.77 & 4.79 & 3.71 & 3.07 & 2.61 & 2.3 \\
\hline & 1.5 & 10.87 & 9.81 & 7.75 & 5.84 & 4.55 & 3.66 & 3.04 & 2.64 & 2.32 \\
\hline & 1.75 & 6.48 & 6.23 & 5.65 & 4.8 & 4.05 & 3.44 & 2.98 & 2.61 & 2.32 \\
\hline & 2 & 4.75 & 4.66 & 4.35 & 3.99 & 3.55 & 3.16 & 2.81 & 2.51 & 2.29 \\
\hline & 0.25 & 3.48 & 3.49 & 3.48 & 3.48 & 3.4 & 2.98 & 2.55 & 2 & 2 \\
\hline & 0.5 & 7.08 & 7.06 & 6.76 & 5.27 & 3.89 & 3.06 & 2.53 & 2.09 & 2 \\
\hline & 0.75 & 31.25 & 24.8 & 10.18 & 5.71 & 3.98 & 3.1 & 2.56 & 2.19 & 2.01 \\
\hline$h=5.05$ & 1 & 251.87 & 36.65 & 10.32 & 5.74 & 4.05 & 3.13 & 2.58 & 2.25 & 2.02 \\
\hline \multirow[t]{7}{*}{$n=4$} & 1.25 & 22.2 & 15.82 & 8.84 & 5.61 & 4.06 & 3.17 & 2.63 & 2.27 & 2.03 \\
\hline & 1.5 & 8.3 & 7.65 & 6.19 & 4.82 & 3.83 & 3.13 & 2.64 & 2.29 & 2.04 \\
\hline & 1.75 & 5.12 & 4.99 & 4.54 & 4 & 3.43 & 2.96 & 2.57 & 2.27 & 2.04 \\
\hline & 2 & 3.8 & 3.72 & 3.56 & 3.33 & 3 & 2.7 & 2.42 & 2.19 & 1.98 \\
\hline & 0.25 & 2.96 & 2.96 & 2.95 & 2.96 & 2.91 & 2.79 & 2.03 & 2 & 2 \\
\hline & 0.5 & 5.63 & 5.63 & 5.5 & 4.51 & 3.42 & 2.75 & 2.17 & 2.01 & 1.99 \\
\hline & 0.75 & 21.45 & 17.95 & 8.46 & 4.96 & 3.5 & 2.74 & 2.26 & 2.04 & 1.93 \\
\hline$h=5.05$ & 1 & 249.09 & 30.01 & 8.77 & 5.02 & 3.54 & 2.78 & 2.32 & 2.06 & 1.88 \\
\hline \multirow[t]{4}{*}{$n=5$} & 1.25 & 18.1 & 13.25 & 7.55 & 4.91 & 3.57 & 2.83 & 2.37 & 2.08 & 1.86 \\
\hline & 1.5 & 6.9 & 6.43 & 5.37 & 4.29 & 3.42 & 2.79 & 2.39 & 2.09 & 1.84 \\
\hline & 1.75 & 4.34 & 4.25 & 3.93 & 3.5 & 3.06 & 2.63 & 2.31 & 2.04 & 1.83 \\
\hline & 2 & 3.26 & 3.24 & 3.08 & 2.89 & 2.64 & 2.42 & 2.18 & 1.96 & 1.8 \\
\hline
\end{tabular}

and develop a new statistic that determines the highest value of the four above-mentioned statistics. This new statistic is represented by $N_{i}$ defined as:

$$
N_{i}=\max \left(C_{i}^{+}, C_{i}^{-}, S_{i}^{+}, S_{i}^{-}\right) \text {. }
$$

Statistic $N_{i}$ is always positive and it has only the upper control limit, say $h$. Whenever $N_{i}$ exceeds control limit $h$, then we say that the process is in an out-ofcontrol state; otherwise, it is in a good state. The ARL results of Max-CUSUM control charting schemes through simulation approach are provided in Table 2. Max-CUSUM chart is sensitive to small shifts in the process mean and dispersion when the sample size increases.

\subsection{SS-EWMA chart}

SS-EWMA was introduced by Xie [14]. Based on the statistics defined in Eqs. (3) and (4), SS-EWMA statistic is constructed and given below in the form of Eq. (12):

$$
\mathrm{SS}_{i}=U_{i}^{2}+V_{i}^{2} \quad \text { for } \quad i=1,2 .
$$

$\mathrm{SS}_{i}$ follows a chi-square distribution when it is divided by $\sigma_{U_{i}}^{2}$, since $\frac{U_{i}}{\sigma_{U_{i}}}$ and $\frac{V_{i}}{\sigma_{V_{i}}}$ are independent and identical standard normal distribution. Based on the information of Eq. (13), the expected value and the variance of $\mathrm{SS}_{i}$ are given by:

$$
\begin{aligned}
& \frac{\mathrm{SS} 1_{i}}{\sigma_{U_{i}}^{2}}=\frac{U_{i}^{2}}{\sigma_{U_{i}}^{2}}+\frac{V_{i}^{2}}{\sigma_{U_{i}}^{2}} \sim \chi_{2}^{2}, \\
& E\left(\mathrm{SS}_{i}\right)=2 \sigma_{U_{i}}^{2}, \\
& \operatorname{Var}\left(\mathrm{SS} 1_{i}\right)=4 \sigma_{U_{i}}^{4} .
\end{aligned}
$$

Since $\mathrm{SS}_{i}$ is always positive, it has only the UCL, given by:

$$
\mathrm{UCL}=E\left(\mathrm{SS}_{i}\right)+L \sqrt{\operatorname{Var}\left(\mathrm{SS}_{i}\right)} .
$$

Since $\sigma_{U_{i}}^{2}=\frac{\lambda}{2-\lambda}$ for the steady case and by the 
Table 3. ARL values for SS-EWMA control charting schemes at $\mathrm{ARL}_{0}=250$.

\begin{tabular}{|c|c|c|c|c|c|c|c|c|c|c|}
\hline & & & & & $a$ & & & & & \\
\hline & $b$ & 0 & 0.25 & 0.5 & 0.75 & 1 & 1.25 & 1.5 & 1.75 & 2 \\
\hline \multirow{8}{*}{$\begin{array}{l}\lambda=0.1 \\
n=5 \\
L=3.6\end{array}$} & 0.25 & 3.47 & 3.38 & 3.17 & 2.97 & 2.78 & 2.28 & 2.01 & 2 & 2 \\
\hline & 0.5 & 6.41 & 5.9 & 4.88 & 4.01 & 3.31 & 2.89 & 2.46 & 2.07 & 2 \\
\hline & 0.75 & 19.98 & 13.24 & 7.51 & 5.08 & 3.84 & 3.13 & 2.66 & 2.24 & 2.04 \\
\hline & 1 & 252.32 & 25.67 & 9.14 & 5.56 & 4.06 & 3.23 & 2.7 & 2.34 & 2.1 \\
\hline & 1.25 & 17.26 & 12.28 & 7.41 & 5.09 & 3.88 & 3.17 & 2.66 & 2.34 & 2.11 \\
\hline & 1.5 & 7.24 & 6.62 & 5.37 & 4.33 & 3.52 & 2.95 & 2.58 & 2.29 & 2.09 \\
\hline & 1.75 & 4.75 & 4.54 & 4.08 & 3.58 & 3.13 & 2.73 & 2.43 & 2.2 & 2.02 \\
\hline & 2 & 3.58 & 3.5 & 3.3 & 3.06 & 2.76 & 2.5 & 2.28 & 2.09 & 1.93 \\
\hline \multirow{8}{*}{$\begin{aligned} \lambda & =0.2 \\
n & =3 \\
L & =4.067\end{aligned}$} & 0.25 & 4.75 & 4.53 & 4.04 & 3.49 & 3.03 & 2.71 & 2.21 & 2 & 2 \\
\hline & 0.5 & 13.18 & 10.93 & 7.32 & 5.15 & 3.86 & 3.13 & 2.63 & 2.22 & 2.03 \\
\hline & 0.75 & 83.33 & 38.27 & 12.93 & 6.66 & 4.49 & 3.43 & 2.8 & 2.38 & 2.12 \\
\hline & 1 & 247.78 & 51.3 & 13.92 & 7.03 & 4.67 & 3.53 & 2.89 & 2.45 & 2.17 \\
\hline & 1.25 & 28.08 & 18.32 & 10.09 & 6.16 & 4.41 & 3.47 & 2.83 & 2.44 & 2.16 \\
\hline & 1.5 & 9.87 & 8.87 & 6.75 & 5.08 & 3.98 & 3.23 & 2.76 & 2.4 & 2.13 \\
\hline & 1.75 & 5.84 & 5.55 & 4.88 & 4.14 & 3.48 & 2.98 & 2.57 & 2.29 & 2.06 \\
\hline & 2 & 4.18 & 4.1 & 3.83 & 3.43 & 3.06 & 2.7 & 2.43 & 2.16 & 1.98 \\
\hline \multirow{8}{*}{$\begin{aligned} \lambda & =0.2 \\
n & =4 \\
L & =4.067\end{aligned}$} & 0.25 & 3.46 & 3.36 & 3.1 & 2.82 & 2.45 & 2.06 & 2 & 2 & 1.97 \\
\hline & 0.5 & 7.96 & 6.94 & 5.26 & 3.96 & 3.16 & 2.62 & 2.18 & 2.01 & 1.98 \\
\hline & 0.75 & 43.93 & 22.49 & 9.1 & 5.22 & 3.69 & 2.89 & 2.39 & 2.1 & 1.96 \\
\hline & 1 & 246.36 & 39.45 & 10.8 & 5.69 & 3.9 & 3 & 2.48 & 2.15 & 1.94 \\
\hline & 1.25 & 21.42 & 14.19 & 7.87 & 5.1 & 3.72 & 2.94 & 2.46 & 2.14 & 1.9 \\
\hline & 1.5 & 7.58 & 6.83 & 5.36 & 4.14 & 3.33 & 2.76 & 2.35 & 2.06 & 1.85 \\
\hline & 1.75 & 4.62 & 4.39 & 3.93 & 3.36 & 2.91 & 2.51 & 2.22 & 1.97 & 1.78 \\
\hline & 2 & 3.39 & 3.27 & 3.1 & 2.82 & 2.55 & 2.28 & 2.07 & 1.88 & 1.71 \\
\hline
\end{tabular}

substitution of Eqs. (14) and (15) into Eq. (16), the UCL approaches asymptotically to:

$$
\begin{aligned}
\mathrm{UCL} & =2 \sigma_{U_{i}}^{2}(1+L), \\
\mathrm{UCL} & =\frac{2 \lambda}{2-\lambda}(1+L) .
\end{aligned}
$$

The ARL results of SS-EWMA control charting schemes are provided in Table 3. SS-EWMA chart is very sensitive to small shifts at both process mean and dispersion when the sample size increases and also when the value of $\lambda$ decreases.

\subsection{SS-CUSUM chart}

Thaga [16] introduced the structure of SS-CUSUM chart, and it is based on the statistics given in Eqs. (7)(10). Let $M_{i}$ and $V_{i}$ be the maximum values of CUSUM statistics that monitor process mean and standard deviation, respectively:

$$
\begin{aligned}
& M_{i}=\max \left(C_{i}^{+}, C_{i}^{-}\right), \\
& V_{i}=\max \left(S_{i}^{+}, S_{i}^{-}\right) .
\end{aligned}
$$

SS-CUSUM is the sum of square of the maximum values of CUSUM statistics that monitor both process mean and standard deviation, and it is given by:

$$
\mathrm{SS} 2_{i}=M_{i}^{2}+V_{i}^{2} \text {. }
$$

$\mathrm{SS} 2 i$ is plotted against the control limit, say $h$. The ARL results of SS-CUSUM control charting schemes for different sample sizes are provided in Table 4. SSCUSUM chart is highly sensitive to small shifts at both process mean and dispersion when the sample size increases.

\section{The proposed control charts}

In this section, we propose four new memory-type control charts that monitor both process mean and 
Table 4. ARL values for SS-CUSUM control charting schemes at $\mathrm{ARL}_{0}=250$ for $k=0.50$.

\begin{tabular}{|c|c|c|c|c|c|c|c|c|c|c|}
\hline & \multicolumn{10}{|c|}{$a$} \\
\hline & $b$ & 0 & 0.25 & 0.5 & 0.75 & 1 & 1.25 & 1.5 & 1.75 & 2 \\
\hline & 0.25 & 4.77 & 4.77 & 4.57 & 4.05 & 3.49 & 3.01 & 2.77 & 2.19 & 2 \\
\hline & 0.5 & 10.82 & 10.61 & 8.44 & 5.94 & 4.37 & 3.48 & 2.94 & 2.52 & 2.13 \\
\hline & 0.75 & 61.21 & 40.17 & 12.99 & 6.96 & 4.81 & 3.7 & 3.03 & 2.59 & 2.26 \\
\hline$n=3$ & 1 & 248.62 & 48.25 & 13.25 & 7.15 & 4.9 & 3.77 & 3.1 & 2.63 & 2.32 \\
\hline \multirow[t]{7}{*}{$h=28.03$} & 1.25 & 27.85 & 19.22 & 10.7 & 6.58 & 4.74 & 3.74 & 3.06 & 2.64 & 2.34 \\
\hline & 1.5 & 10.19 & 9.24 & 7.25 & 5.55 & 4.34 & 3.52 & 3.01 & 2.61 & 2.32 \\
\hline & 1.75 & 6.14 & 5.87 & 5.24 & 4.51 & 3.82 & 3.27 & 2.83 & 2.52 & 2.26 \\
\hline & 2 & 4.44 & 4.38 & 4.08 & 3.72 & 3.33 & 2.96 & 2.66 & 2.38 & 2.16 \\
\hline & 0.25 & 3.61 & 3.61 & 3.48 & 3.16 & 2.88 & 2.5 & 2.04 & 2 & 2 \\
\hline & 0.5 & 7.4 & 7.21 & 6.07 & 4.59 & 3.59 & 2.96 & 2.46 & 2.08 & 2 \\
\hline & 0.75 & 32.31 & 23.68 & 9.67 & 5.62 & 4 & 3.14 & 2.6 & 2.23 & 2.03 \\
\hline$n=4$ & 1 & 248.93 & 36.44 & 10.52 & 5.88 & 4.13 & 3.2 & 2.65 & 2.29 & 2.07 \\
\hline \multirow[t]{7}{*}{$h=27.9$} & 1.25 & 21.47 & 14.81 & 8.45 & 5.49 & 4.02 & 3.18 & 2.65 & 2.3 & 2.07 \\
\hline & 1.5 & 7.87 & 7.25 & 5.83 & 4.55 & 3.65 & 3.02 & 2.57 & 2.25 & 2.02 \\
\hline & 1.75 & 4.88 & 4.7 & 4.27 & 3.68 & 3.2 & 2.75 & 2.42 & 2.17 & 1.96 \\
\hline & 2 & 3.63 & 3.55 & 3.34 & 3.07 & 2.79 & 2.52 & 2.27 & 2.07 & 1.88 \\
\hline & 0.25 & 3.06 & 3.05 & 2.96 & 2.77 & 2.39 & 2.03 & 2 & 2 & 1.98 \\
\hline & 0.5 & 5.83 & 5.75 & 4.94 & 3.86 & 3.11 & 2.6 & 2.12 & 2 & 1.99 \\
\hline & 0.75 & 22.22 & 16.89 & 7.95 & 4.84 & 3.5 & 2.79 & 2.31 & 2.05 & 1.95 \\
\hline$n=5$ & 1 & 250.48 & 29.45 & 8.81 & 5.07 & 3.62 & 2.86 & 2.37 & 2.11 & 1.92 \\
\hline \multirow[t]{4}{*}{$h=27.9$} & 1.25 & 17.32 & 12.52 & 7.17 & 4.74 & 3.54 & 2.82 & 2.38 & 2.09 & 1.89 \\
\hline & 1.5 & 6.58 & 6.05 & 5 & 3.99 & 3.2 & 2.68 & 2.32 & 2.04 & 1.84 \\
\hline & 1.75 & 4.18 & 4.05 & 3.67 & 3.21 & 2.82 & 2.47 & 2.18 & 1.97 & 1.77 \\
\hline & 2 & 3.13 & 3.08 & 2.92 & 2.7 & 2.46 & 2.23 & 2.02 & 1.84 & 1.69 \\
\hline
\end{tabular}

variance simultaneously. The proposed charts are based on the usual mean standardization of $\bar{X}$ for the location and a three-parameter logarithmic transformation to $S^{2}$ (cf., [17]) for the dispersion statistic. This section is divided into four parts for the four proposals discussed below.

It is to be mentioned that we have used the Monte Carlo simulation approach to evaluate ARL measures provided for this study. We have performed these simulations by developing a code in $R$ language and executing it a reasonable number of times, say $10^{4}$ times (for a relevant discussion on the number of simulations needed in control charting studies, one may see $[18])$.

\subsection{Sum of square exponentially weighted moving average variance control chart}

Sum of Square Exponentially Weighted Moving Average Variance (SS-EWMAVAR) is a new control chart that monitors both process mean and variance simultaneously. It is constructed by replacing the statistic that monitors the process dispersion in SS-EWMA control chart with the three-parameter logarithmic transformation to $S^{2}[17]$.

Suppose that $X_{i j} \sim N\left(\mu, \sigma^{2}\right)$ for $i=1,2,3, \cdots$ and $j=1,2,3, \cdots, n$, where $n$ is the sample size. Let $\bar{X}_{i}=\left(X_{i 1}+\cdots+X_{i n}\right) / n$ and $S_{i}^{2}=\frac{\sum_{j=1}^{n}\left(X_{i j}-\bar{X}\right)^{2}}{n-1}$ be the sample mean and variance of the distribution, respectively. These two statistics are the minimum variance unbiased estimators of $\mu_{0}$ and $\sigma_{0}^{2}$, respectively. $\bar{X}_{i}$ is standardized into normal distribution and given by:

$$
G_{i}=\sqrt{n} \frac{\left(\bar{X}_{i}-\mu_{0}\right)}{\sigma_{0}} \sim N(0,1) .
$$

Castagliola [17] used the three-parameter logarithmic transformation in order to monitor the process variance, which is given below by:

$$
T_{i}=a+b \ln \left(S_{i}^{2}+c\right),
$$

where $a, b$, and, $c$ are constants greater than zero and are defined below by:

$$
\begin{aligned}
& b=B(n), \quad c=C(n) \sigma_{0}^{2}, \\
& a=A(n)-2 B(n) \ln \left(\sigma_{0}\right),
\end{aligned}
$$

where $A(n), B(n)$, and $C(n)$ are functions that depend on the value of sample size $(n)$. Table 5 reproduces 
Table 5. Values of $A(n), B(n), C(n), \mu_{T}(n)$ and $\sigma_{T}(n)$ counterparts.

\begin{tabular}{cccccc}
\hline $\boldsymbol{n}$ & $\boldsymbol{A}(\boldsymbol{n})$ & $\boldsymbol{B}(\boldsymbol{n})$ & $\boldsymbol{C}(\boldsymbol{n})$ & $\boldsymbol{\mu}_{\boldsymbol{T}}(\boldsymbol{n})$ & $\boldsymbol{\sigma}_{\boldsymbol{T}}(\boldsymbol{n})$ \\
\hline 3 & -0.6627 & 1.8136 & 0.6777 & 0.02472 & 0.9165 \\
4 & -0.7882 & 2.1089 & 0.6261 & 0.01266 & 0.9502 \\
5 & -0.8969 & 2.3647 & 0.5979 & 0.00748 & 0.967 \\
6 & -0.994 & 2.5941 & 0.5801 & 0.00485 & 0.9765 \\
7 & -1.0827 & 2.8042 & 0.5678 & 0.00335 & 0.9825 \\
8 & -1.1647 & 2.9992 & 0.5588 & 0.00243 & 0.9864 \\
9 & -1.2413 & 3.182 & 0.5519 & 0.00182 & 0.9892 \\
10 & -1.3135 & 3.3548 & 0.5465 & 0.00141 & 0.9912 \\
11 & -1.382 & 3.5189 & 0.5421 & 0.00112 & 0.9927 \\
12 & -1.4473 & 3.6757 & 0.5384 & 0.0009 & 0.9938 \\
13 & -1.5097 & 3.826 & 0.5354 & 0.00074 & 0.9947 \\
14 & -1.5697 & 3.9705 & 0.5327 & 0.00062 & 0.9955 \\
15 & -1.6275 & 4.11 & 0.5305 & 0.00052 & 0.996 \\
\hline & & & & &
\end{tabular}

the values of $A(n), B(n), C(n), \mu_{T}(n)$, and $\sigma_{T}(n)$ for sample sizes 3 to 15 .

In order to monitor the process variance, we will use distribution $T_{i}$ and standardize it as normal so as to have the same distribution with $G_{i}, F_{i}=\frac{\left(T_{i}-\bar{\mu}_{T}(n)\right)}{\sigma_{T}(n)} \sim$ $N(0,1)$.

Now, $G_{i}$ and $F_{i}$ are transformed into EWMA statistics as follows:

$$
\begin{gathered}
U_{i}=(1-\lambda) U_{i-1}+\lambda G_{i}, \\
V_{i}=(1-\lambda) V_{i-1}+\lambda F_{i} .
\end{gathered}
$$

The initial values of $U_{i}$ and $V_{i}$ are set to be $U_{0}$ and $V_{0}$, respectively, and $0<\lambda \leq 1$. Based on the statistics in Eqs. (23) and (24), SS-EWMA statistic is constructed in Eq. (25):

$$
\mathrm{SSEW}_{i}=U_{i}^{2}+V_{i}^{2} \quad \text { for } \quad i=1,2,3, \cdots
$$

It has been proved in Section 2.3 that $\mathrm{SSEW}_{i}$ follows a chi-square distribution; their expected value and the variance are given by $E\left(\mathrm{SSEW}_{i}\right)=2 \sigma_{U_{i}}^{2}$ and $\operatorname{Var}\left(\mathrm{SSEW}_{i}\right)=4 \sigma_{U_{i}}^{4}$, respectively.

Since $\mathrm{SSEW}_{i}$ is always positive, it has only the Upper Control Limit (UCL). Now, UCL is given by:

$$
\mathrm{UCL}=E\left(\mathrm{SSEW}_{i}\right)+L \sqrt{\operatorname{Var}\left(\mathrm{SSEW}_{i}\right)} .
$$

By the substitution of $E\left(\mathrm{SSEW}_{i}\right)$ and $V\left(\mathrm{SSEW}_{i}\right)$ in Eq. (26), we can simplify UCL as: UCL $=2 \sigma_{U_{i}}^{2}(1+L)$. Since $\sigma_{U_{i}}^{2}=\frac{\lambda}{2-\lambda}$ for the steady case, UCL approaches asymptotically to:

$$
\mathrm{UCL}=\frac{2 \lambda}{2-\lambda}(1+L) .
$$

ARL results of SS-EWMAVAR control chart, through simulation, for various shifts in the process mean $(a)$ and dispersion $(b)$ are provided in Table 6 . We observed that SS-EWMAVAR chart is very sensitive to small shifts in the process dispersion when the value of $\lambda$ increases.

\subsection{Maximum exponentially weighted moving average variance control chart}

Maximum Exponentially Weighted Moving Average Variance (Max-EWMAVAR) is a new control chart for monitoring both process mean and variance simultaneously. It is constructed by replacing the statistic that monitors the process dispersion in Max-EWMA control chart with the three-parameter logarithmic transformation to $S^{2}$.

Under the same assumption in Section 3.1, the statistics of Eqs. (23) and (24) can be combined in another form as:

$$
\mathrm{MEW}_{i}=\max \left(\left|U_{i}\right|,\left|V_{i}\right|\right)
$$

$\mathrm{MEW}_{i}$ is plotted against the Upper Control Limit (UCL) derived below:

$$
\mathrm{UCL}=E\left(\mathrm{MEW}_{i}\right)+L \sqrt{\operatorname{Var}\left(\mathrm{MEW}_{i}\right)} .
$$

Therefore, $\mathrm{UCL}=\sigma_{U_{i}}(1.128379+L 0.602811)$ :

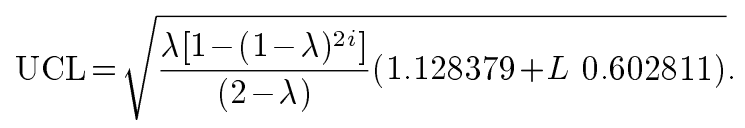

UCL will approach steady value when $i$ gets larger. Consequently:

$$
\mathrm{UCL}=\sqrt{\frac{\lambda}{(2-\lambda)}}(1.128379+L 0.602811) .
$$

ARL results of Max-EWMAVAR control chart, through simulation, for various shifts in process mean (a) and dispersion (b) are provided in Table 7 for varying $\lambda$. In addition, Table 8 provides ARL values at $\lambda=0.2$ for some selective $n$. The result of its sensitive time-varying control limit is given in Table 9 for various sample size $(n)$. We observed that MAX-EWMAVAR chart is very sensitive to small shifts for the process dispersion when the value of $\lambda$ decreases.

\subsection{Sum of square of cumulative sum variance control chart}

Sum of square cumulative sum variance (SSCUSUMVAR) is a new control chart for monitoring both process mean and variance simultaneously. SSCUSUMVAR statistic is constructed by replacing the statistic that monitors the process dispersion in SSCUSUM control chart with the three-parameter logarithmic transformation to $S^{2}$. 
Table 6. ARL values for SS-EWMAVAR control charting schemes at $\mathrm{ARL}_{0}=250$.

\begin{tabular}{|c|c|c|c|c|c|c|c|c|c|c|}
\hline & \multicolumn{10}{|c|}{$a$} \\
\hline & $b$ & 0 & 0.25 & 0.5 & 0.75 & 1 & 1.25 & 1.5 & 1.75 & 2 \\
\hline & 0.25 & 4.73 & 4.36 & 4.01 & 3.4 & 3 & 2.93 & 2.07 & 2 & 2 \\
\hline & 0.5 & 7.05 & 6.42 & 5.18 & 4.14 & 3.37 & 2.95 & 2.49 & 2.07 & 2 \\
\hline & 0.75 & 20.67 & 13.29 & 7.56 & 5.06 & 3.82 & 3.12 & 2.65 & 2.24 & 2.04 \\
\hline$L=3.55$ & 1 & 250.57 & 25.29 & 9.05 & 5.53 & 4.04 & 3.22 & 2.7 & 2.32 & 2.1 \\
\hline \multirow[t]{7}{*}{$\lambda=0.10$} & 1.25 & 16.37 & 11.87 & 7.35 & 5.07 & 3.88 & 3.13 & 2.65 & 2.31 & 2.1 \\
\hline & 1.5 & 7.12 & 6.47 & 5.28 & 4.23 & 3.49 & 2.94 & 2.56 & 2.26 & 2.07 \\
\hline & 1.75 & 4.68 & 4.48 & 4.05 & 3.54 & 3.08 & 2.71 & 2.44 & 2.2 & 2 \\
\hline & 2 & 3.58 & 3.5 & 3.3 & 3.05 & 2.75 & 2.5 & 2.28 & 2.09 & 1.94 \\
\hline & 0.25 & 4.03 & 3.91 & 3.18 & 2.99 & 2.37 & 2 & 2 & 2 & 1.94 \\
\hline & 0.5 & 6.79 & 5.97 & 4.54 & 3.47 & 2.83 & 2.27 & 2.02 & 1.99 & 1.87 \\
\hline & 0.75 & 35.12 & 16.48 & 7.28 & 4.42 & 3.19 & 2.54 & 2.14 & 1.98 & 1.82 \\
\hline$L=3.99$ & 1 & 250.41 & 30.99 & 8.74 & 4.83 & 3.38 & 2.64 & 2.21 & 1.97 & 1.77 \\
\hline \multirow[t]{7}{*}{$\lambda=0.20$} & 1.25 & 15.75 & 11.16 & 6.5 & 4.31 & 3.23 & 2.58 & 2.17 & 1.92 & 1.71 \\
\hline & 1.5 & 6.1 & 5.53 & 4.43 & 3.52 & 2.87 & 2.42 & 2.1 & 1.84 & 1.66 \\
\hline & 1.75 & 3.84 & 3.67 & 3.34 & 2.9 & 2.52 & 2.21 & 1.97 & 1.77 & 1.58 \\
\hline & 2 & 2.89 & 2.85 & 2.69 & 2.48 & 2.22 & 2.02 & 1.82 & 1.67 & 1.54 \\
\hline & 0.25 & 3.87 & 3.52 & 3.04 & 2.67 & 2.02 & 2 & 2 & 1.7 & 1.05 \\
\hline & 0.5 & 8.06 & 6.6 & 4.5 & 3.25 & 2.52 & 2.08 & 1.96 & 1.73 & 1.33 \\
\hline & 0.75 & 83.44 & 26.21 & 8.11 & 4.28 & 2.93 & 2.3 & 1.97 & 1.73 & 1.45 \\
\hline$L=4.15$ & 1 & 250.16 & 38.91 & 9.41 & 4.68 & 3.13 & 2.39 & 1.99 & 1.71 & 1.47 \\
\hline \multirow[t]{7}{*}{$\lambda=0.30$} & 1.25 & 15.88 & 11.2 & 6.33 & 4.02 & 2.94 & 2.31 & 1.93 & 1.65 & 1.45 \\
\hline & 1.5 & 5.72 & 5.13 & 4.08 & 3.21 & 2.58 & 2.16 & 1.84 & 1.61 & 1.43 \\
\hline & 1.75 & 3.47 & 3.29 & 2.99 & 2.57 & 2.23 & 1.95 & 1.72 & 1.55 & 1.38 \\
\hline & 2 & 2.54 & 2.49 & 2.36 & 2.18 & 1.95 & 1.76 & 1.59 & 1.46 & 1.35 \\
\hline & 0.25 & 4.99 & 4.19 & 3.13 & 2.27 & 2 & 1.92 & 1.25 & 1 & 1 \\
\hline & 0.5 & 66.4 & 20.67 & 6.43 & 3.4 & 2.35 & 1.93 & 1.56 & 1.18 & 1.03 \\
\hline & 1 & 248.39 & 55.81 & 12.99 & 4.98 & 2.94 & 2.19 & 1.69 & 1.38 & 1.17 \\
\hline$L=4.24$ & 1.25 & 17.54 & 12.04 & 6.44 & 3.95 & 2.74 & 2.04 & 1.67 & 1.41 & 1.23 \\
\hline \multirow[t]{3}{*}{$\lambda=0.50$} & 1.5 & 5.34 & 5 & 3.86 & 2.98 & 2.26 & 1.92 & 1.59 & 1.36 & 1.22 \\
\hline & 1.75 & 3.04 & 2.94 & 2.55 & 2.28 & 1.97 & 1.7 & 1.5 & 1.34 & 1.22 \\
\hline & 2 & 2.22 & 2.22 & 2.03 & 1.86 & 1.64 & 1.56 & 1.4 & 1.27 & 1.19 \\
\hline
\end{tabular}

Following the inspiration of Section 3.1, statistics $G_{i}$ and $F_{i}$ are defined in the same way. The two statistics are integrated into CUSUM statistics to monitor the process mean and variance as given in Eqs. (30)-(33):

$$
\begin{aligned}
\mathrm{SSC}_{i}^{+} & =\max \left[0, G_{i}-k+\mathrm{SSC}_{i-1}^{+}\right] \\
\mathrm{SSC}_{i}^{-} & =\max \left[0,-G_{i}-k+\mathrm{SSC}_{i-1}^{-}\right] \\
\mathrm{SSS}_{i}^{+} & =\max \left[0, F_{i}-k+\mathrm{SSS}_{i-1}^{+}\right] \\
\mathrm{SSS}_{i}^{-} & =\max \left[0,-F_{i}-k+\mathrm{SSS}_{i-1}^{-}\right]
\end{aligned}
$$

where $\mathrm{SSC}_{0}=\mathrm{SSS}_{0}=0$ are the starting points.
Let $M C_{i}$ and $V S_{i}$ be the maximum values of CUSUM statistics that monitor process mean and variance, respectively:

$$
\begin{aligned}
& M C_{i}=\max \left(\mathrm{SSC}_{i}^{+}, \mathrm{SSC}_{i}^{-}\right), \\
& V S_{i}=\max \left(\mathrm{SSS}_{i}^{+}, \mathrm{SSS}_{i}^{-}\right) .
\end{aligned}
$$

SS-CUSUMVAR is the sum of square of the maximum values of CUSUM statistics that monitor both process mean and variance and it is given by variance, respectively:

$$
\mathrm{SS}_{i}=M C_{i}^{2}+V S_{i}^{2}
$$


Table 7. ARL values for Max-EWMAVAR control charting scheme when $n=5$ at $\mathrm{ARL}_{0}=250$.

\begin{tabular}{|c|c|c|c|c|c|c|c|c|c|c|}
\hline & \multicolumn{10}{|c|}{$a$} \\
\hline & $b$ & 0 & 0.25 & 0.5 & 0.75 & 1 & 1.25 & 1.5 & 1.75 & 2 \\
\hline & 0.25 & 4.13 & 4.13 & 4.13 & 4.13 & 3.82 & 3 & 2.55 & 2 & 2 \\
\hline & 0.5 & 6.47 & 6.51 & 6.32 & 5.03 & 3.75 & 3.02 & 2.52 & 2.06 & 2 \\
\hline & 0.75 & 18.91 & 16.07 & 8.53 & 5.22 & 3.78 & 3.03 & 2.53 & 2.16 & 2.02 \\
\hline$L=2.77$ & 1 & 250.29 & 24.54 & 8.73 & 5.26 & 3.84 & 3.07 & 2.55 & 2.24 & 2.03 \\
\hline \multirow[t]{7}{*}{$\lambda=0.1$} & 1.25 & 14.06 & 10.99 & 7.08 & 4.98 & 3.78 & 3.06 & 2.58 & 2.25 & 2.05 \\
\hline & 1.5 & 5.65 & 5.39 & 4.76 & 4.04 & 3.42 & 2.93 & 2.53 & 2.26 & 2.03 \\
\hline & 1.75 & 3.69 & 3.62 & 3.42 & 3.22 & 2.94 & 2.63 & 2.38 & 2.17 & 2 \\
\hline & 2 & 2.82 & 2.79 & 2.75 & 2.63 & 2.49 & 2.34 & 2.19 & 2.04 & 1.89 \\
\hline & 0.25 & 3.659 & 3.66 & 3.67 & 3.65 & 3.03 & 2.33 & 2 & 2 & 1.95 \\
\hline & 0.5 & 6.051 & 6.09 & 5.8 & 4.36 & 3.14 & 2.41 & 2.04 & 1.97 & 1.8 \\
\hline & 0.75 & 29.09 & 22.5 & 8.55 & 4.6 & 3.17 & 2.47 & 2.09 & 1.92 & 1.71 \\
\hline$L=2.99$ & 1 & 251.18 & 30.54 & 8.43 & 4.62 & 3.23 & 2.53 & 2.13 & 1.89 & 1.67 \\
\hline \multirow[t]{7}{*}{$\lambda=0.2$} & 1.25 & 15.51 & 11.4 & 6.59 & 4.35 & 3.22 & 2.56 & 2.14 & 1.86 & 1.65 \\
\hline & 1.5 & 5.53 & 5.14 & 4.39 & 3.62 & 2.93 & 2.45 & 2.11 & 1.85 & 1.65 \\
\hline & 1.75 & 3.46 & 3.4 & 3.15 & 2.83 & 2.54 & 2.25 & 1.99 & 1.8 & 1.61 \\
\hline & 2 & 2.59 & 2.57 & 2.49 & 2.34 & 2.18 & 2 & 1.84 & 1.68 & 1.56 \\
\hline & 0.25 & 3.251 & 3.26 & 3.25 & 3.24 & 2.89 & 2.03 & 2 & 1.85 & 1.1 \\
\hline & 0.5 & 6.649 & 6.72 & 6.27 & 4.25 & 2.87 & 2.17 & 1.95 & 1.68 & 1.27 \\
\hline & 0.75 & 59.94 & 41.7 & 10.1 & 4.51 & 2.92 & 2.25 & 1.91 & 1.64 & 1.34 \\
\hline$L=3.06$ & 1 & 251.4 & 38.3 & 9 & 4.46 & 2.98 & 2.29 & 1.89 & 1.63 & 1.38 \\
\hline \multirow[t]{5}{*}{$\lambda=0.3$} & 1.25 & 16.22 & 11.9 & 6.51 & 4.12 & 2.96 & 2.31 & 1.9 & 1.61 & 1.41 \\
\hline & 1.5 & 5.429 & 5 & 4.17 & 3.36 & 2.65 & 2.2 & 1.87 & 1.62 & 1.43 \\
\hline & 1.75 & 3.276 & 3.19 & 2.94 & 2.58 & 2.29 & 2.01 & 1.76 & 1.58 & 1.42 \\
\hline & 2 & 2.388 & 2.38 & 2.28 & 2.13 & 1.96 & 1.79 & 1.64 & 1.5 & 1.38 \\
\hline & 1 & 250.5 & 89.6 & 21.2 & 7.01 & 3.34 & 2.06 & 1.49 & 1.22 & 1.08 \\
\hline$L=3.12$ & 1.25 & 21.88 & 16.7 & 9.11 & 4.83 & 3.01 & 2.04 & 1.54 & 1.28 & 1.13 \\
\hline \multirow[t]{3}{*}{$\lambda=0.8$} & 1.5 & 6.205 & 5.62 & 4.39 & 3.31 & 2.41 & 1.89 & 1.54 & 1.31 & 1.18 \\
\hline & 1.75 & 3.175 & 3.08 & 2.74 & 2.34 & 1.98 & 1.68 & 1.47 & 1.3 & 1.19 \\
\hline & 2 & 2.131 & 2.11 & 2 & 1.83 & 1.67 & 1.5 & 1.37 & 1.25 & 1.17 \\
\hline
\end{tabular}

The control limit $(h)$ of $\mathrm{SS}_{i}$ is derived through simulation. Statistic $\mathrm{SS}_{i}$ is plotted against $h$. For outof-control signals, we compare the statistic with the corresponding control limit and receive the signals if points fall outside the limit, i.e. $\mathrm{SS}_{i}>h$.

\subsection{Maximum cumulative sum variance control chart}

Maximum cumulative sum variance (Max-CUSUMVAR) is a new control chart that monitors both process mean and variance simultaneously. It is based on the assumption in Section 3.3. The statistics of Eqs. (34) and (35) can be combined in another form as:

$$
\operatorname{MCVAR}_{i}=\max \left(M C_{i}, V S_{i}\right) .
$$

Since statistic MCVAR $_{i}$ is always positive, it has the only upper control limit $h$. Whenever $\operatorname{MCVAR}_{i}$ exceeds control limit $h$, then we say that the process is in an out-of-control state; otherwise, it is statistically in-control.

\section{Comparative analysis}

In this section, we will compare the performance of the proposed charts with their counterparts including max-chart, Max-CUSUM, Max-EWMA, SS-CUSUM, and SS-EWMA control charts. For different amounts of shifts in process mean $(a)$ and dispersion $(b)$, ARL values of the proposed charts and the other competing charts are provided in Tables 1-11. These results are based on $10^{4}$ Monte Carlo simulations, at each run, for our study purposes. We have created Figures 1-6 
Table 8. ARL values for Max-EWMAVAR control charting scheme for $\lambda=0.2$ at $\mathrm{ARL}_{0}=250$.

\begin{tabular}{lcccccccccc}
\hline & & & \multicolumn{1}{c}{$\boldsymbol{a}$} \\
\hline & $\boldsymbol{b}$ & $\mathbf{0}$ & $\mathbf{0 . 2 5}$ & $\mathbf{0 . 5}$ & $\mathbf{0 . 7 5}$ & $\mathbf{1}$ & $\mathbf{1 . 2 5}$ & $\mathbf{1 . 5}$ & $\mathbf{1 . 7 5}$ & $\mathbf{2}$ \\
\hline & 0.25 & 6.37 & 6.37 & 6.38 & 6.11 & 4.28 & 3.15 & 2.77 & 2.04 & 2 \\
& 0.5 & 15.04 & 15.14 & 12.63 & 6.91 & 4.36 & 3.27 & 2.66 & 2.19 & 2.02 \\
$\boldsymbol{n}=\mathbf{3}$ & 0.75 & 194.05 & 88.97 & 15.85 & 6.89 & 4.44 & 3.33 & 2.69 & 2.29 & 2.06 \\
$\boldsymbol{L}=\mathbf{3 . 0 2}$ & 1 & 256.08 & 50.26 & 13.43 & 6.73 & 4.48 & 3.38 & 2.77 & 2.35 & 2.08 \\
& 1.25 & 24.56 & 17.6 & 10.13 & 6.17 & 4.39 & 3.42 & 2.78 & 2.39 & 2.1 \\
& 1.5 & 9.26 & 8.51 & 6.79 & 5.21 & 4.09 & 3.29 & 2.78 & 2.4 & 2.12 \\
& 1.75 & 5.63 & 5.45 & 4.86 & 4.23 & 3.62 & 3.09 & 2.66 & 2.35 & 2.09 \\
& 2 & 4.14 & 4.09 & 3.85 & 3.51 & 3.17 & 2.81 & 2.52 & 2.26 & 2.04 \\
& & & & & & & & & & \\
$\boldsymbol{n}=\mathbf{4}$ & 0.25 & 5.41 & 5.4 & 5.4 & 5.32 & 4.18 & 3.1 & 2.69 & 2.03 & 2 \\
& 0.5 & 10.63 & 10.66 & 9.77 & 6.44 & 4.27 & 3.2 & 2.62 & 2.17 & 2.01 \\
& 0.75 & 64.24 & 47.25 & 14.34 & 6.63 & 4.32 & 3.25 & 2.65 & 2.26 & 2.04 \\
& 1 & 249.27 & 48.04 & 13 & 6.56 & 4.39 & 3.31 & 2.71 & 2.32 & 2.06 \\
& 1.25 & 27.94 & 18.67 & 10 & 6.2 & 4.34 & 3.35 & 2.74 & 2.35 & 2.07 \\
& 1.5 & 9.97 & 8.95 & 6.93 & 5.17 & 4.06 & 3.27 & 2.74 & 2.35 & 2.07 \\
& 1.75 & 5.9 & 5.66 & 5.02 & 4.25 & 3.61 & 3.05 & 2.65 & 2.32 & 2.06 \\
& 2 & 4.29 & 4.2 & 3.89 & 3.55 & 3.18 & 2.82 & 2.52 & 2.26 & 2.04 \\
\hline
\end{tabular}

Table 9. ARL values for time-varying Max-EWMAVAR control charting scheme for $\lambda=0.2$ at $\mathrm{ARL}_{0}=250$.

\begin{tabular}{|c|c|c|c|c|c|c|c|c|c|c|}
\hline & \multicolumn{10}{|c|}{$a$} \\
\hline & $b$ & 0 & 0.25 & 0.5 & 0.75 & 1 & 1.25 & 1.5 & 1.75 & 2 \\
\hline & 0.25 & 5.82 & 5.83 & 5.83 & 5.52 & 3.54 & 2.37 & 1.93 & 1.38 & 1.02 \\
\hline & 0.5 & 14.87 & 14.98 & 12.33 & 6.31 & 3.6 & 2.44 & 1.85 & 1.45 & 1.16 \\
\hline & 0.75 & 201.33 & 90.77 & 15.29 & 6.13 & 3.62 & 2.49 & 1.87 & 1.5 & 1.25 \\
\hline$n=3$ & 1 & 258.08 & 49.15 & 12.41 & 5.81 & 3.59 & 2.52 & 1.95 & 1.57 & 1.33 \\
\hline \multirow{7}{*}{$L=3.034$} & 1.25 & 22.46 & 15.69 & 8.68 & 5.04 & 3.4 & 2.5 & 1.93 & 1.6 & 1.4 \\
\hline & 1.5 & 7.48 & 6.8 & 5.32 & 3.93 & 3.01 & 2.33 & 1.92 & 1.63 & 1.42 \\
\hline & 1.75 & 4.15 & 4.01 & 3.57 & 3.05 & 2.52 & 2.11 & 1.81 & 1.6 & 1.43 \\
\hline & 2 & 2.87 & 2.83 & 2.66 & 2.4 & 2.15 & 1.9 & 1.7 & 1.53 & 1.4 \\
\hline & 0.25 & 4.79 & 4.79 & 4.8 & 4.7 & 3.42 & 2.3 & 1.89 & 1.31 & 1.01 \\
\hline & 0.5 & 10.31 & 10.33 & 9.39 & 5.82 & 3.5 & 2.37 & 1.8 & 1.41 & 1.14 \\
\hline & 0.75 & 65.34 & 47.81 & 13.73 & 5.88 & 3.49 & 2.41 & 1.84 & 1.48 & 1.23 \\
\hline$n=4$ & 1 & 252.78 & 46.86 & 11.95 & 5.62 & 3.5 & 2.46 & 1.88 & 1.53 & 1.31 \\
\hline \multirow[t]{7}{*}{$L=3.7$} & 1.25 & 25.5 & 16.62 & 8.53 & 4.99 & 3.34 & 2.44 & 1.91 & 1.58 & 1.38 \\
\hline & 1.5 & 8.11 & 7.2 & 5.42 & 3.93 & 2.98 & 2.33 & 1.9 & 1.6 & 1.4 \\
\hline & 1.75 & 4.36 & 4.14 & 3.68 & 3.08 & 2.54 & 2.1 & 1.81 & 1.58 & 1.42 \\
\hline & 2 & 3 & 2.88 & 2.69 & 2.42 & 2.17 & 1.9 & 1.71 & 1.55 & 1.41 \\
\hline & 0.25 & 2.9221 & 2.9227 & 2.9166 & 2.9056 & 2.1741 & 1.7056 & 1.041 & 1 & 1 \\
\hline & 0.5 & 5.3571 & 5.4056 & 5.0906 & 3.534 & 2.2981 & 1.6298 & 1.1968 & 1.0256 & 1.0014 \\
\hline & 0.75 & 28.505 & 21.799 & 7.7832 & 3.7448 & 2.302 & 1.6674 & 1.2964 & 1.0931 & 1.0203 \\
\hline$n=5$ & 1 & 248.06 & 29.297 & 7.4438 & 3.7105 & 2.3807 & 1.7319 & 1.3683 & 1.17 & 1.0627 \\
\hline \multirow[t]{4}{*}{$L=2.992$} & 1.25 & 14.414 & 10.39 & 5.7319 & 3.4203 & 2.3373 & 1.7474 & 1.4202 & 1.2226 & 1.1073 \\
\hline & 1.5 & 4.9141 & 4.4422 & 3.5428 & 2.7191 & 2.1149 & 1.7225 & 1.4279 & 1.2589 & 1.1368 \\
\hline & 1.75 & 2.7985 & 2.6746 & 2.4348 & 2.1242 & 1.8047 & 1.5673 & 1.388 & 1.2618 & 1.1485 \\
\hline & 2 & 1.9793 & 1.9561 & 1.8515 & 1.7418 & 1.5625 & 1.4376 & 1.3099 & 1.2253 & 1.1528 \\
\hline
\end{tabular}


Table 10. ARL values for SS-CUSUMVAR charting schemes at $\mathrm{ARL}_{0}=250, h=27.66$, and $k=0.5$.

\begin{tabular}{lcccccccccc}
\hline & $\boldsymbol{b}$ & $\mathbf{0}$ & $\mathbf{0 . 2 5}$ & $\mathbf{0 . 5}$ & $\mathbf{0 . 7 5}$ & $\mathbf{1}$ & $\mathbf{1 . 2 5}$ & $\mathbf{1 . 5}$ & $\mathbf{1 . 7 5}$ & $\mathbf{2}$ \\
\hline & 0.25 & 6.71 & 6.7 & 6.19 & 5.04 & 4.04 & 3.21 & 2.99 & 2.43 & 2.01 \\
& 0.5 & 12.16 & 11.85 & 9.2 & 6.19 & 4.53 & 3.55 & 2.99 & 2.56 & 2.14 \\
$\boldsymbol{n}=\mathbf{3}$ & 0.75 & 73.53 & 46.09 & 13.38 & 7.06 & 4.85 & 3.71 & 3.04 & 2.6 & 2.26 \\
$\boldsymbol{h}=\mathbf{2 8}$ & 1 & 252.22 & 47.87 & 13.33 & 7.19 & 4.92 & 3.77 & 3.08 & 2.65 & 2.32 \\
& 1.25 & 24.36 & 17.79 & 9.96 & 6.48 & 4.69 & 3.67 & 3.07 & 2.63 & 2.33 \\
& 1.5 & 9.41 & 8.56 & 6.91 & 5.32 & 4.24 & 3.49 & 2.94 & 2.58 & 2.3 \\
& 1.75 & 5.72 & 5.51 & 5.03 & 4.33 & 3.7 & 3.2 & 2.81 & 2.48 & 2.22 \\
& 2 & 4.27 & 4.19 & 3.93 & 3.61 & 3.24 & 2.91 & 2.62 & 2.37 & 2.17 \\
& & & & & & & & & & \\
$\boldsymbol{n}=\mathbf{4}$ & 0.25 & 5.04 & 5.03 & 4.62 & 3.97 & 3.1 & 2.97 & 2.2 & 2 & 2 \\
$\boldsymbol{h}=\mathbf{2 7 . 6}$ & 0.5 & 8.24 & 8.04 & 6.55 & 4.8 & 3.68 & 3 & 2.51 & 2.09 & 2 \\
& 0.75 & 34.52 & 24.64 & 9.84 & 5.65 & 4.01 & 3.15 & 2.6 & 2.23 & 2.03 \\
& 1.25 & 252.72 & 36.63 & 10.38 & 5.8 & 4.11 & 3.2 & 2.64 & 2.28 & 2.05 \\
& 1.5 & 7.49 & 14.09 & 8.23 & 5.38 & 3.96 & 3.14 & 2.64 & 2.29 & 2.05 \\
& 1.75 & 4.73 & 4.58 & 4.15 & 3.64 & 3.15 & 2.75 & 2.42 & 2.17 & 1.97 \\
& 2 & 3.57 & 3.48 & 3.3 & 3.06 & 2.77 & 2.5 & 2.26 & 2.06 & 1.88 \\
& & & & & & & & & & \\
& 0.25 & 4.05 & 4.05 & 3.97 & 3.14 & 2.99 & 2.28 & 2 & 2 & 2 \\
$\boldsymbol{n}=\mathbf{5}$ & 0.5 & 6.52 & 6.39 & 5.32 & 4.07 & 3.2 & 2.67 & 2.13 & 2 & 1.99 \\
$\boldsymbol{h}=\mathbf{2 7 . 6 6}$ & 1.75 & 23.06 & 17.48 & 8.07 & 4.88 & 3.51 & 2.79 & 2.3 & 2.05 & 1.96 \\
& 1.25 & 249.9 & 29.13 & 8.85 & 5.12 & 3.62 & 2.84 & 2.37 & 2.09 & 1.92 \\
& 1.5 & 6.44 & 11.98 & 7.03 & 4.75 & 3.5 & 2.8 & 2.37 & 2.08 & 1.88 \\
& 1.75 & 4.15 & 4.02 & 3.63 & 3.21 & 2.8 & 2.46 & 2.17 & 1.96 & 1.77 \\
& 2 & 3.14 & 3.08 & 2.91 & 2.7 & 2.48 & 2.23 & 2.03 & 1.85 & 1.69 \\
\hline & & & & & & & & & &
\end{tabular}

Table 11. ARL values for Max-CUSUMVAR control charting scheme at $\mathrm{ARL}_{0}=250$ and $k=0.5$.

\begin{tabular}{lcccccccccc}
\hline & & & & & $\boldsymbol{a}$ & & & & \\
\hline & $\boldsymbol{b}$ & $\mathbf{0}$ & $\mathbf{0 . 2 5}$ & $\mathbf{0 . 5}$ & $\mathbf{0 . 7 5}$ & $\mathbf{1}$ & $\mathbf{1 . 2 5}$ & $\mathbf{1 . 5}$ & $\mathbf{1 . 7 5}$ & $\mathbf{2}$ \\
\hline & 0.25 & 6.44 & 6.43 & 6.44 & 6.25 & 4.63 & 3.56 & 3 & 2.49 & 2.01 \\
& 0.5 & 11.66 & 11.61 & 10.56 & 6.9 & 4.7 & 3.57 & 2.96 & 2.5 & 2.11 \\
$\boldsymbol{h}=\mathbf{5 . 0 6}$ & 0.75 & 69.22 & 48.15 & 13.77 & 7.06 & 4.77 & 3.64 & 2.97 & 2.52 & 2.21 \\
$\boldsymbol{n}=\mathbf{3}$ & 1 & 248.73 & 47.71 & 13.24 & 7.09 & 4.83 & 3.7 & 3.01 & 2.58 & 2.27 \\
& 1.25 & 25.44 & 18.77 & 10.39 & 6.65 & 4.75 & 3.7 & 3.07 & 2.61 & 2.3 \\
& 1.5 & 9.9 & 9.06 & 7.33 & 5.64 & 4.46 & 3.62 & 3.03 & 2.63 & 2.32 \\
& 1.75 & 6.05 & 5.86 & 5.36 & 4.62 & 3.94 & 3.39 & 2.94 & 2.59 & 2.31 \\
& 2 & 4.53 & 4.46 & 4.18 & 3.86 & 3.47 & 3.11 & 2.79 & 2.5 & 2.28 \\
& & & & & & & & & & \\
$\boldsymbol{h}=\mathbf{5 . 0 3}$ & 0.25 & 4.87 & 4.88 & 4.87 & 4.84 & 3.91 & 3.01 & 2.54 & 2 & 2 \\
$\boldsymbol{n}=\mathbf{4}$ & 0.5 & 7.93 & 7.9 & 7.53 & 5.51 & 3.91 & 3.06 & 2.51 & 2.08 & 2 \\
& 0.75 & 33.27 & 26.12 & 10.36 & 5.71 & 3.97 & 3.09 & 2.55 & 2.18 & 2.01 \\
& 1.25 & 252.77 & 36.58 & 10.29 & 5.73 & 4.04 & 3.12 & 2.58 & 2.24 & 2.01 \\
& 1.5 & 7.88 & 7.31 & 5.99 & 4.73 & 3.78 & 3.11 & 2.63 & 2.28 & 2.04 \\
& 1.75 & 4.98 & 4.84 & 4.44 & 3.93 & 3.39 & 2.94 & 2.56 & 2.27 & 2.03 \\
& 2 & 3.76 & 3.68 & 3.51 & 3.3 & 2.99 & 2.7 & 2.42 & 2.2 & 1.99 \\
& & & & & & & & & & \\
& 0.25 & 4.01 & 4.01 & 4.02 & 4.01 & 3.35 & 2.89 & 2.03 & 2 & 2 \\
$\boldsymbol{h}=\mathbf{5 . 0 3 5}$ & 0.5 & 6.28 & 6.29 & 6.1 & 4.73 & 3.44 & 2.75 & 2.17 & 2.01 & 1.98 \\
$\boldsymbol{n}=\mathbf{5}$ & 1 & 250.21 & 29.25 & 8.77 & 5.04 & 3.56 & 2.78 & 2.32 & 2.05 & 1.88 \\
& 1.25 & 17.09 & 12.7 & 7.43 & 4.91 & 3.58 & 2.82 & 2.37 & 2.06 & 1.85 \\
& 1.5 & 6.7 & 6.38 & 5.3 & 4.23 & 3.39 & 2.78 & 2.37 & 2.08 & 1.86 \\
& 1.75 & 4.32 & 4.24 & 3.89 & 3.47 & 3.02 & 2.64 & 2.31 & 2.05 & 1.84 \\
& 2 & 3.28 & 3.22 & 3.09 & 2.9 & 2.69 & 2.42 & 2.19 & 1.99 & 1.8 \\
\hline & & & & & & & & & &
\end{tabular}




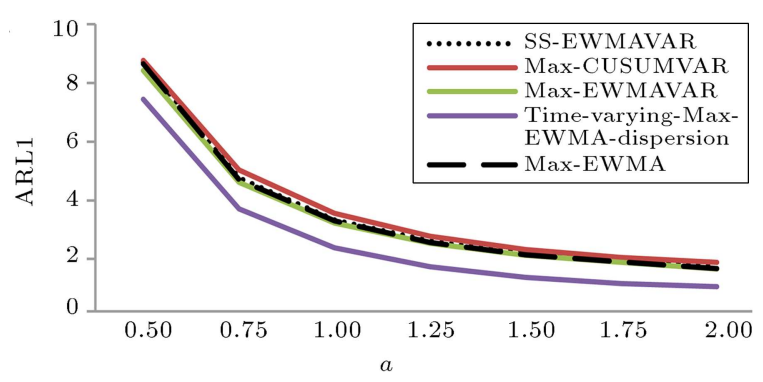

Figure 1. ARL curves for the process mean of the proposed charts versus Max-EWMA at $\mathrm{ARL}_{0}=250$, $b=1$, and $\lambda=0.20$.

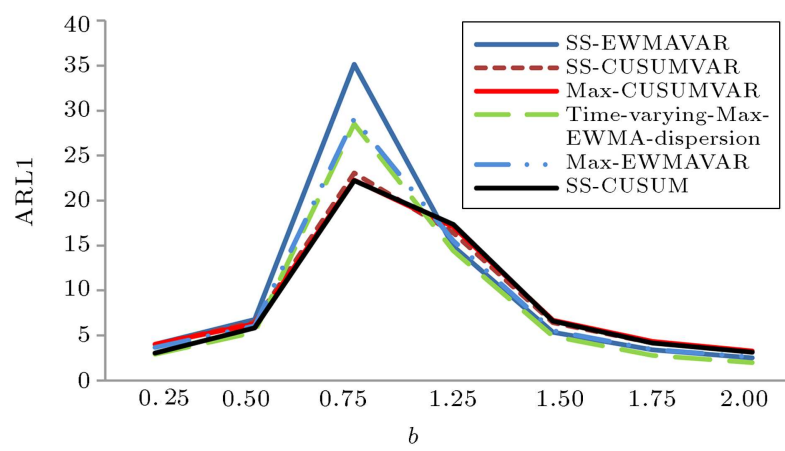

Figure 2. ARL curves for the process dispersion of the proposed charts versus Max-EWMA when $a=0$ and $\lambda=0.20$.

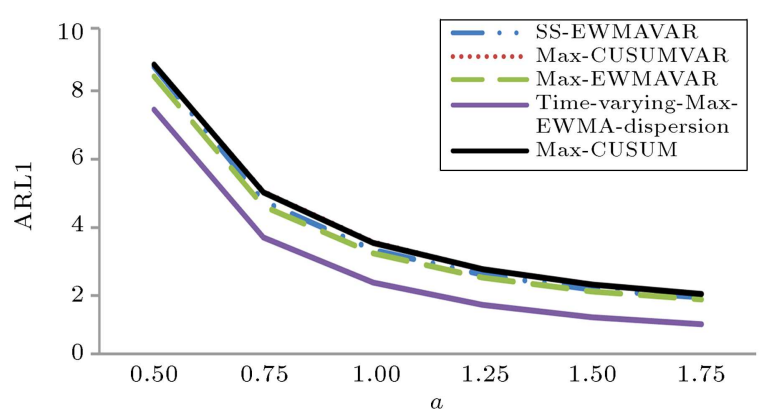

Figure 3. ARL curves for the process mean of the proposed charts versus Max-CUSUM at $\mathrm{ARL}_{0}=250$, $b=1$, and $\lambda=0.20$.

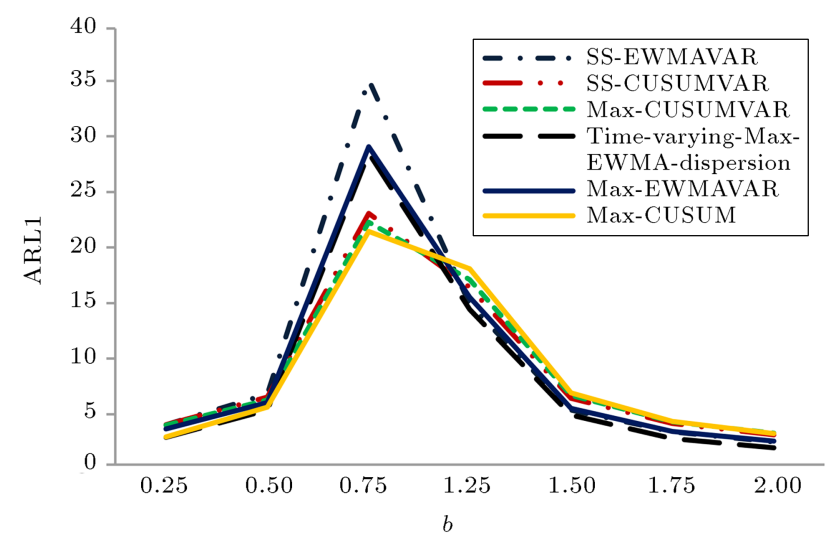

Figure 4. ARL curves for the process dispersion of the proposed charts versus Max-CUSUM when, $a=0$ and $\lambda=0.20$

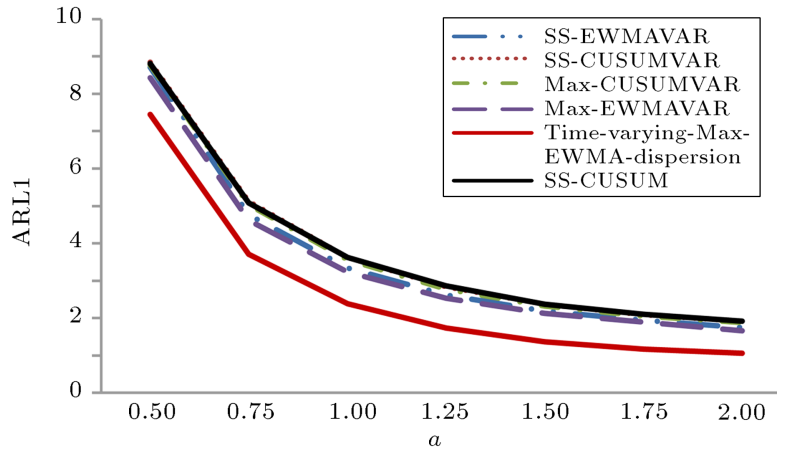

Figure 5. ARL curves for the process mean of the proposed charts versus SS-CUSUM at $\mathrm{ARL}_{0}=250, b=1$, and $\lambda=0.20$.

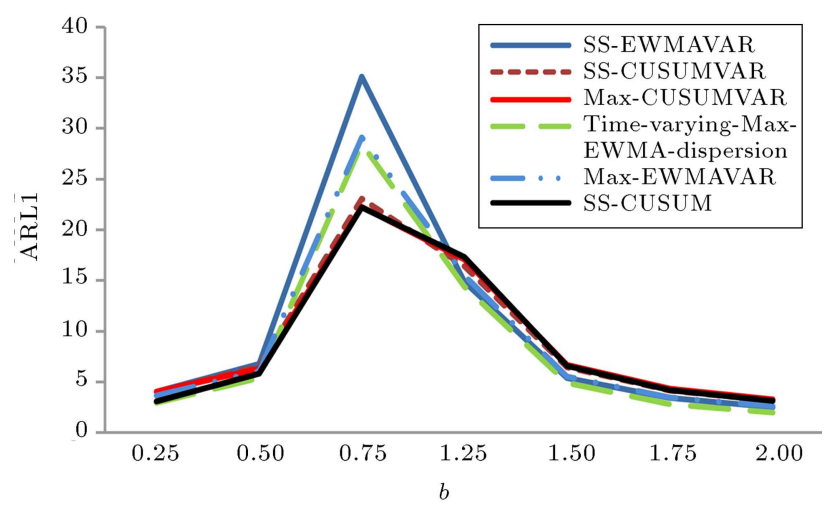

Figure 6. ARL Curves for the process dispersion of the proposed charts versus SS-CUSUM at $a=0$ and $\lambda=0.20$.

to serve purpose of ease in comparison and discussion. For a comparative analysis of the proposed charts with their existing counterparts, these results show that:

- The proposed charts are better than Max-CUSUM, Max-EWMA, SS-CUSUM, and SS-EWMA in the increasing shifts of the process dispersion when $n=3$;

- For $n=4$, only SS-CUSUMVAR and MaxCUSUMVAR are better than their counterparts under study at the increasing shifts of the process dispersion;

- SS-EWMAVAR and Max-EWMAVAR are better than Max-EWMA and SS-EWMA in the increasing shifts of the process dispersion when $n=5$ for all values of $\lambda$;

- SS-EWMAVAR and Max-EWMAVAR are better than Max-CUSUM and SS-CUSUM at the increasing shifts of the process dispersion when $n=5$;

- SS-EWMAVAR is better than Max-CUSUM, SS-CUSUM, and SS-EWMA at a very small shift $(a<0.5)$ of the process mean when $n=5$;

- Max-EWMAVAR is better than Max-CUSUM, and SS-CUSUM at small shift $(a<0.5)$ of the process 
mean, but slightly better than SS-EWMA at all shifts of the mean when $n=5$;

- SS-EWMAVAR, Max-EWMAVAR, and Max-EWMA have nearly equal values at all shifts in the process mean when $n=5$;

- SS-CUSUMVAR and Max-CUSUMVAR similar ARL values with Max-CUSUM, Max-EWMA, SS-CUSUM, and SS-EWMA at all shifts in the process mean when $n=5$;

- SS-CUSUMVAR is slightly better than the performance of all its existing counterparts (except max-chart) at all shifts of the process dispersion when $n=5$;

- Max-CUSUMVAR is slightly better than the performance of all its existing counterparts in small and moderate shifts of the process dispersion when $n=5$;

- SS-EWMAVAR, SS-CUSUMVAR, Max-CUSUMVAR, and Max-EWMAVAR are performing better than max-chart at small and moderate shifts in both parameters $(n=5)$;

- Generally, the proposed charts are poorer at detecting decreasing shifts in the process dispersion than their counterparts.

\section{Illustrative example}

A practical example was taken from the book of Montgomery [19] about the hard-bake process with photolithography in semiconductor manufacturing. Twenty-five samples were initially used in phase 1 with the subgroup, each of 5 sample size of wafers, and they are all in a statistical process. Another set of twenty samples of wafers are added to be monitored in phase 2. The output of the proposed charts and their counterparts are shown in Table 12 and their graphical displays are shown in Figures 7 to 12. The sample mean and variance are calculated to be 1.53184 and

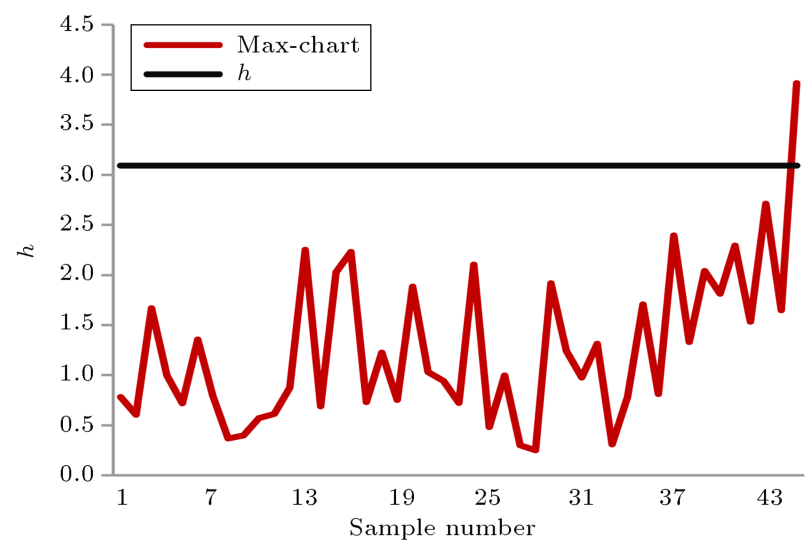

Figure 7. Max-chart when $h=3.09$ at $\mathrm{ARL}_{0}=250$.

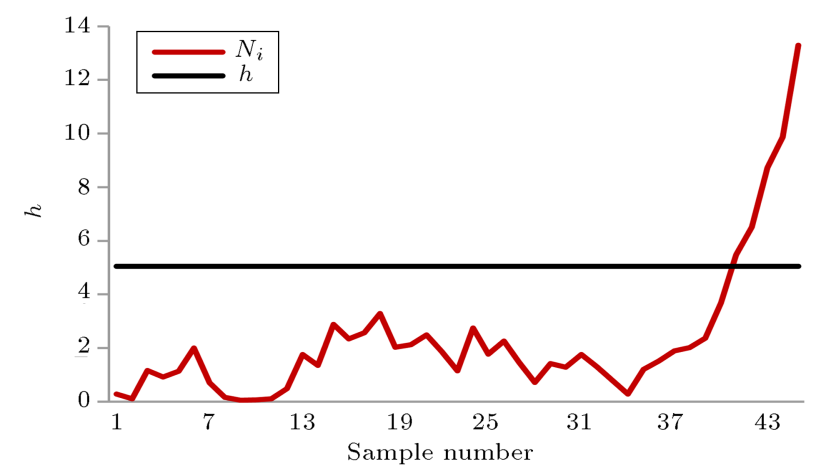

Figure 8. Max-CUSUM when $h=5.05, k=0.5$ at $\mathrm{ARL}_{0}$ $=250$.

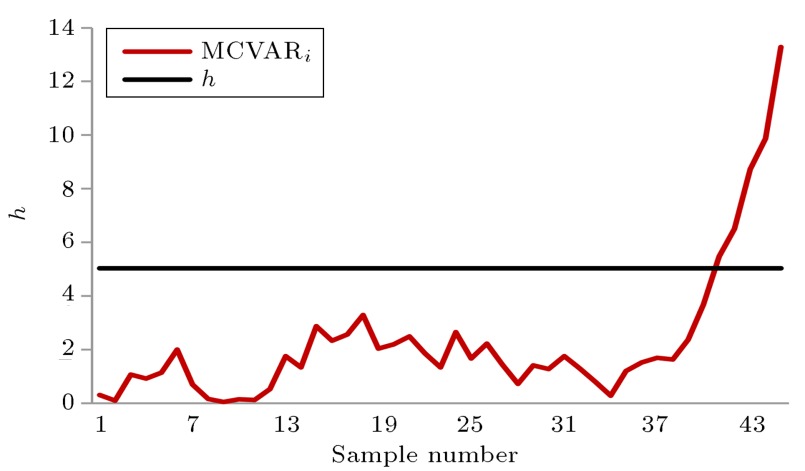

Figure 9. Max-CUSUMVAR when $h=5.035$ at $\mathrm{ARL}_{0}=$ 250.

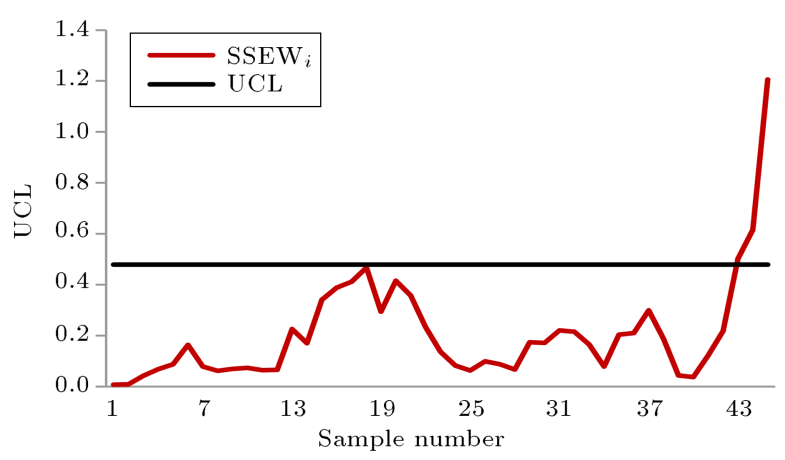

Figure 10. SS-EWMAVAR when $L=3.55$, and $\lambda=0.1$ at $\mathrm{ARL}_{0}=250$.

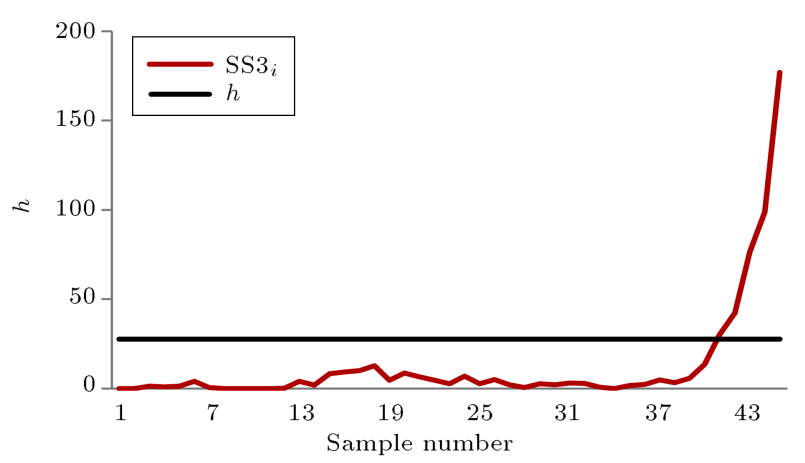

Figure 11. SS-CUSUMVAR when $h=27.66$ at $\mathrm{ARL}_{0}=$ 250. 
Table 12. Output of the hard-bake process for the proposed charts and their counterparts.

\begin{tabular}{|c|c|c|c|c|c|c|c|c|c|}
\hline$S /$ no & $N_{i}$ & $M_{i}$ & $\mathrm{SS1}_{i}$ & SSEW $_{i}$ & $\mathrm{SS3}_{i}$ & $\operatorname{MCVAR}_{i}$ & MaxChat & $\mathrm{SS2}_{i}$ & $\mathrm{MEW}_{i}$ \\
\hline 1 & 0.28 & 0.08 & 0.01 & 0.01 & 0.1 & 0.31 & 0.78 & 0.08 & 0.081 \\
\hline 2 & 0.1 & 0.09 & 0.01 & 0.01 & 0.01 & 0.1 & 0.6 & 0.01 & 0.09 \\
\hline 3 & 1.17 & 0.16 & 0.04 & 0.04 & 1.33 & 1.07 & 1.67 & 1.55 & 0.163 \\
\hline 4 & 0.92 & 0.25 & 0.07 & 0.07 & 0.94 & 0.92 & 1 & 0.99 & 0.247 \\
\hline 5 & 1.14 & 0.29 & 0.09 & 0.09 & 1.3 & 1.14 & 0.72 & 1.3 & 0.294 \\
\hline 6 & 2 & 0.4 & 0.16 & 0.16 & 4 & 2 & 1.36 & 4 & 0.4 \\
\hline 7 & 0.7 & 0.28 & 0.08 & 0.08 & 0.56 & 0.7 & 0.8 & 0.55 & 0.28 \\
\hline 8 & 0.16 & 0.25 & 0.06 & 0.06 & 0.03 & 0.16 & 0.37 & 0.03 & 0.248 \\
\hline 9 & 0.06 & 0.26 & 0.07 & 0.07 & 0 & 0.06 & 0.4 & 0 & 0.263 \\
\hline 10 & 0.07 & 0.27 & 0.07 & 0.07 & 0.02 & 0.15 & 0.57 & 0 & 0.267 \\
\hline 11 & 0.11 & 0.25 & 0.06 & 0.06 & 0.02 & 0.12 & 0.61 & 0.01 & 0.253 \\
\hline 12 & 0.49 & 0.23 & 0.07 & 0.07 & 0.28 & 0.53 & 0.87 & 0.24 & 0.233 \\
\hline 13 & 1.75 & 0.44 & 0.23 & 0.23 & 4.03 & 1.75 & 2.25 & 3.88 & 0.435 \\
\hline 14 & 1.35 & 0.4 & 0.17 & 0.17 & 1.88 & 1.35 & 0.69 & 1.85 & 0.401 \\
\hline 15 & 2.88 & 0.56 & 0.34 & 0.34 & 8.3 & 2.88 & 2.03 & 8.3 & 0.564 \\
\hline 16 & 2.33 & 0.5 & 0.39 & 0.39 & 9.36 & 2.33 & 2.23 & 8.99 & 0.503 \\
\hline 17 & 2.57 & 0.53 & 0.41 & 0.41 & 9.99 & 2.57 & 0.73 & 9.69 & 0.526 \\
\hline 18 & 3.29 & 0.6 & 0.47 & 0.47 & 12.77 & 3.29 & 1.22 & 12.66 & 0.596 \\
\hline 19 & 2.04 & 0.46 & 0.3 & 0.29 & 4.68 & 2.04 & 0.75 & 4.7 & 0.461 \\
\hline 20 & 2.13 & 0.46 & 0.42 & 0.42 & 8.69 & 2.2 & 1.89 & 8.39 & 0.457 \\
\hline 21 & 2.49 & 0.51 & 0.36 & 0.36 & 6.65 & 2.49 & 1.03 & 6.63 & 0.515 \\
\hline 22 & 1.87 & 0.45 & 0.24 & 0.23 & 4.59 & 1.87 & 0.94 & 4.36 & 0.451 \\
\hline 23 & 1.15 & 0.36 & 0.14 & 0.14 & 2.65 & 1.34 & 0.72 & 2.17 & 0.361 \\
\hline 24 & 2.75 & 0.26 & 0.09 & 0.08 & 7.05 & 2.65 & 2.1 & 7.57 & 0.264 \\
\hline 25 & 1.77 & 0.25 & 0.07 & 0.06 & 2.8 & 1.67 & 0.48 & 3.12 & 0.247 \\
\hline 26 & 2.26 & 0.27 & 0.1 & 0.1 & 4.95 & 2.23 & 1 & 5.13 & 0.275 \\
\hline 27 & 1.46 & 0.28 & 0.09 & 0.09 & 2.09 & 1.45 & 0.3 & 2.14 & 0.276 \\
\hline 28 & 0.71 & 0.25 & 0.07 & 0.07 & 0.52 & 0.72 & 0.25 & 0.51 & 0.247 \\
\hline 29 & 1.42 & 0.41 & 0.17 & 0.17 & 2.66 & 1.42 & 1.92 & 2.56 & 0.414 \\
\hline 30 & 1.28 & 0.41 & 0.17 & 0.17 & 2.22 & 1.28 & 1.24 & 2.19 & 0.409 \\
\hline 31 & 1.76 & 0.47 & 0.22 & 0.22 & 3.16 & 1.76 & 0.98 & 3.13 & 0.465 \\
\hline 32 & 1.3 & 0.42 & 0.21 & 0.22 & 2.88 & 1.3 & 1.32 & 2.74 & 0.423 \\
\hline 33 & 0.8 & 0.38 & 0.16 & 0.17 & 0.74 & 0.8 & 0.31 & 0.69 & 0.381 \\
\hline 34 & 0.28 & 0.26 & 0.08 & 0.08 & 0.08 & 0.28 & 0.78 & 0.08 & 0.265 \\
\hline 35 & 1.21 & 0.41 & 0.2 & 0.2 & 1.84 & 1.21 & 1.71 & 1.78 & 0.409 \\
\hline 36 & 1.52 & 0.45 & 0.21 & 0.21 & 2.4 & 1.52 & 0.81 & 2.39 & 0.45 \\
\hline 37 & 1.9 & 0.47 & 0.32 & 0.3 & 4.91 & 1.69 & 2.4 & 6.46 & 0.472 \\
\hline 38 & 2.02 & 0.35 & 0.21 & 0.19 & 3.34 & 1.63 & 1.33 & 4.76 & 0.32 \\
\hline 39 & 2.37 & 0.23 & 0.06 & 0.04 & 5.75 & 2.37 & 2.04 & 6.09 & 0.202 \\
\hline 40 & 3.68 & 0.17 & 0.04 & 0.04 & 13.61 & 3.68 & 1.81 & 13.6 & 0.143 \\
\hline 41 & 5.48 & 0.35 & 0.13 & 0.12 & 30.17 & 5.48 & 2.3 & 30.14 & 0.345 \\
\hline 42 & 6.51 & 0.46 & 0.22 & 0.22 & 42.38 & 6.51 & 1.53 & 42.38 & 0.464 \\
\hline 43 & 8.72 & 0.69 & 0.51 & 0.5 & 76.55 & 8.72 & 2.71 & 76.5 & 0.689 \\
\hline 44 & 9.87 & 0.78 & 0.62 & 0.62 & 98.73 & 9.87 & 1.65 & 98.56 & 0.785 \\
\hline 45 & 13.28 & 1.1 & 1.21 & 1.21 & 176.86 & 13.28 & 3.91 & 176.83 & 1.098 \\
\hline Control limits & 5.05 & 0.644 & 0.484 & 0.479 & 27.6 & 5.035 & 3.09 & 27.9 & 0.642 \\
\hline
\end{tabular}




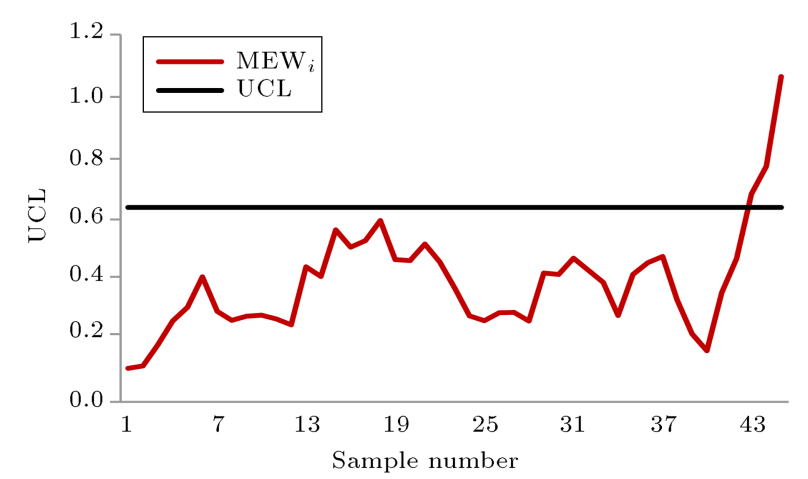

Figure 12. Max-EWMAVAR when $L=2.77$, and $\lambda=0.1$ at $\mathrm{ARL}_{0}=250$.

0.1360771 , respectively. The UCL of Max-EWMA, SSEWMA, SS-EWMAVAR, and Max-EWMAVAR can be calculated from Eqs. (6b), (18), (27), and (29), respectively; we substitute the values of $L$, sample mean and variance in the equations. For a desired incontrol $\mathrm{ARL}_{0}=250$, we fixed the value of $k=0.50$ and $\lambda=0.10$ for each of the charts where necessary; the values of $h$ and $L$ are derived from Tables 1 to 11 .

The results show that:

- There is the increased large values in the process at samples 41 to 45 ;

- SS-CUSUMVAR and Max-CUSUMVAR detect 5 out-of-control signals at sample points 41-45;

- SS-EWMAVAR, Max-EWMAVAR, Max-EWMA, and SS-EWMA triggers 3 out-of control points, at sample points $43-45$;

- Max-chart gives only one out-of-control signal at sample point 45 .

\section{Summary, conclusions and recommendations}

In this study, we have proposed four new control charts that monitor the process mean and dispersion simultaneously. We have investigated ARL properties of the proposed schemes and compared them with the existing counterparts including Max-EWMA, Max-CUSUM, SS-EWMA, and SS-CUSUM. The comparisons showed that the proposed schemes are really performing better for the increasing shifts in the process dispersion than the other existing schemes covered in this study. They have slightly the same ARL values in the process mean with their counterparts. The scope of this study may be extended to monitor process vector mean and dispersion simultaneously in the multivariate setups for an improved and efficient monitoring of process parameters.

\section{Acknowledgements}

The authors are thankful to the anonymous reviewers and the Editor for their constructive comments. The authors are also indebted to King Fahd University of Petroleum and Minerals Dhahran Saudi Arabia for providing excellent research facilities under project FT151001.

\section{Acronyms}

Max-EWMA Max-Exponentially Weighed Moving Average

Max-CUSUM Max-Cumulative Sum

SS-EWMA Sum of Square Exponential

Weighted Moving Average

SS-CUSUM Sum of Square Cumulative Sum

Max-EWMAVAR Maximum Exponentially

Weighted Moving Average Variance

Max-CUSUMVAR Maximum Cumulative Sum

Variance

SS-EWMAVAR Sum of Square Exponentially

Weighted Moving Average Variance

SS-CUSUMVAR Sum of Square Cumulative Sum Variance

SPC Statistical Process Control

CUSUM Cumulative Sum

EWMA Exponential Weighted Moving

Average

UCL Upper Control Limit

$R \quad$ Range

$S^{2} \quad$ Sample Variance

\section{References}

1. Shewhart W., Economic Control of Quality Manufactured Product, D. Van Nostrand, New York; reprinted by the American Society for Quality Control in 1980, Milwauker, WI (1931).

2. Page, E.S. "Continuous inspection schemes", Biometrika, 41, pp. 100-115 (1954).

3. Roberts, S.W. "Control chart tests based on geometric moving averages", Technometrics, 1, pp. 239-250 (1959).

4. Abbasi, S.A. and Miller, A. "On proper choice of variability control chart for normal and non-normal processes", Quality and Reliability Engineering International, 28, pp. 279-296 (2012).

5. Abbas, N., Riaz, M. and Does, R.J.M.M. "Enhancing the performance of EWMA charts", Quality and Reliability Engineering International, 27, pp. 821-833 (2011). 
6. Abbas, N., Riaz, M. and Does, R.J.M.M. "CS-EWMA chart for monitoring process dispersion", Quality and Reliability Engineering International, 29(5), pp. 653663 (2013).

7. Abbas, N., Riaz, M. and Does, R.J.M.M. "Mixed exponentially weighted moving average - cumulative sum charts for process monitoring", Quality and Reliability Engineering International, 29(3), pp. 345-356 (2013).

8. Riaz, M., Abbas, N. and Does, R.J.M.M. "Improving the performance of CUSUM charts", Quality and Reliability Engineering International, 27, pp. 415-424 (2011).

9. Haq, A. "A new hybrid exponentially weighted moving average control chart for monitoring process mean", Quality and Reliability Engineering International, 29, pp. 1015-1025 (2013).

10. Haq, A. "An improved mean deviation exponentially weighted moving average control chart to monitor process dispersion under ranked set sampling", Journal of Statistical Computation and Simulation, 84, pp. 2011-1024 (2014).

11. Haq, A., Brown, J. and Moltchanova, E. "Improved fast initial response features for exponentially weighted moving average and cumulative sum control charts", Quality and Reliability Engineering International, 30(5), pp. 697-710 (2014).

12. Zaman, B., Riaz, M., Abbas, N. and Does, R.J.M.M. "Mixed CUSUM-EWMA control charts: An efficient way of monitoring process location", Quality and Reliability Engineering International, 31(8), pp. 14071421 (2015).

13. Chen, G. and Cheng, S.W. "Max-chart: Combining Xbar chart and S chart", Statistical Sinica, 8, pp. 263271 (1998).

14. Xie, H. "Contributions to qualimetry", Ph.D. Thesis, University of Manitoba, Winnipeg, Canada (1999).
15. Cheng, S.W. and Thaga, K. "The Max-CUSUM chart", Frontiers in Statistical Quality Control, 9 (2010).

16. Thaga, K. "SS-CUSUM chart", Economic Quality Control, 24(1), pp. 117-128 (2009).

17. Castagliola, P. "A new $\mathrm{S}^{2}$-EWMA control chart for monitoring the process variance", Quality and Reliability Engineering International, 21(8), pp. 781-794 (2005).

18. Mundform, D.J., Schaffer, J., Kim, M.J., Shaw, D., Thongteeraparp, A. and Supawan, P. "Number of replications required in Monte Carlo simulation studies: A synthesis of four studies", Journal of Modern Applied Statistical Methods, 10(1), article\#4 (2011).

19. Montgomery, D.C., Introduction to Statistical Quality Control, 6th Ed., John Wiley \& Sons, New York (2009).

\section{Biographies}

Jimoh Olawale Ajadi received his MSc degree in Applied Statistics at King Fahd University of Petroleum \& Minerals (KFUPM), Dhahran, Saudi Arabia. He is currently pursuing his $\mathrm{PhD}$ in the Department of System Engineering and Engineering Management at City University of Hong Kong. His research interest is statistical process control.

Muhammad Riaz holds the position of Professor of Statistics in the Department of Mathematics and Statistics, King Fahd University of Petroleum and Minerals, Dhahran, Saudi Arabia. His current research interests include statistical process control, nonparametric techniques, mathematical statistics, and experimental design. 